\title{
The influence of internal magnetic layers on the frequencies of solar $p$-modes ${ }^{\star}$
}

\author{
C. Foullon ${ }^{1,2, \star \star}$ and B. Roberts ${ }^{1}$ \\ ${ }^{1}$ School of Mathematics and Statistics, University of St. Andrews, Scotland, UK \\ e-mail: foullonc@astro.warwick.ac.uk \\ 2 Center Plasma Astrophysics, K. U. Leuven, Celestijnenlaan 200B, 3001 Heverlee, Belgium
}

Received 26 August 2004 / Accepted 24 April 2005

\begin{abstract}
Buried magnetic fields at the base of the convection zone and in the anchoring zones of sunspots are presumed to vary over the solar activity cycle. Their effect on $p$-mode oscillations is explored in detail, through theoretical modelling. The helioseismic signature from a "tachocline" or "shallow" horizontal layer of magnetic field, buried in a plane-stratified model of the Sun, is explored by examining frequency shifts of various order and degree. $p$-modes propagating perpendicular to the magnetic field lines are found to yield the largest frequency shifts. However, frequency shifts due to buried magnetic fields are considerably smaller than observationally determined shifts over the solar cycle. Nonetheless, an analytical approach to the problem provides useful insight for solar and stellar applications.
\end{abstract}

Key words. Sun: helioseismology - Sun: interior - Sun: magnetic fields - Sun: oscillations

\section{Introduction}

The influence of a magnetic field on $p$-modes may be thought of in several ways (see, e.g., Roberts 2001). With the view that such an influence can explain observed cyclical variations in global mode frequencies (e.g., Libbrecht \& Woodard 1991; Chaplin et al. 1998; Howe et al. 1999), it is of interest to explore how magnetic changes occurring over the solar cycle may induce frequency shifts. Three magnetic regions have been investigated as responsible for the changes: $(a)$ the canopy field of the chromosphere and coronal atmosphere (e.g., Campbell \& Roberts 1989; Evans \& Roberts 1990, 1992; Jain \& Roberts 1994, 1996; Vanlommel 2001); (b) the fibril fields of the photosphere (Bogdan \& Zweibel 1985; Zweibel \& Bogdan 1986); and $(c)$ the strong fields that reside in the convective overshoot region, at the base of the convection zone (Roberts \& Campbell 1986; Campbell 1987; Daniell 1998). A fourth region is now considered: the anchorage zone of sunspots some $50 \mathrm{Mm}$ below the surface.

Magnetic fields, as well as temperature changes, introduce pressure deviations that play a significant role in modulating the resonant frequencies of $p$-mode oscillations. Those pressure deviations occurring in the atmosphere or below the surface of the Sun can explain the frequency shifts observed on the timescale of the solar activity cycle

\footnotetext{
* All appendices are only available in electronic form at http://www. edpsciences.org

$\star \star$ Present address: Department of Physics, University of Warwick, Coventry CV4 7AL, UK.
}

(see, e.g., Campbell \& Roberts 1989; Evans \& Roberts 1990, 1992; Goldreich et al. 1991; Jain \& Roberts 1994). A separate study of the contribution of internal magnetic layers can clarify the relative importance of surface effects.

Results from helioseismology provide realistic constraints for choosing parameters suitable to represent the magnetic layers buried in the solar interior. Important clues are given in particular by the even-index splitting coefficients, which display changes over the solar cycle. Gough \& Thompson (1990) solved the adiabatic oscillation equation in a slowly rotating Sun that includes a toroidal field at the base of the convection zone, and noted that the amplitudes of the even-order splitting coefficients vary as the square of the magnetic field strength. By comparing the theoretical results of Gough \& Thompson (1990) with changes observed by GONG, Basu (1997) deduced that the toroidal field at the base of the convection zone would have a strength not larger than $3 \times 10^{5} \mathrm{G}$. This result was further supported by Antia et al. (2000), who extended the formulation of Gough \& Thompson (1990) by including the perturbation in the gravitational potential and allowing for differential rotation in the interior, and used more recent GONG and MDI data. The value of $3 \times 10^{5} \mathrm{G}$ is slightly above but in broad agreement with the range $3 \times 10^{4}-10^{5} \mathrm{G}$ suggested by numerical simulations of active region emergence and evolution at the solar surface (see, e.g., Choudhuri \& Gilman 1987; Schüssler et al. 1994; Fisher et al. 2000, and references therein). These values are somewhat lower than the estimate of $8 \times 10^{5} \mathrm{G}$ deduced by Roberts \& Campbell (1986) from matching theoretical frequency shifts with observed shifts in low degree modes (Woodard \& Noyes 1985). 
Signatures of a significant perturbation at a shallow depth below the photosphere $(30 \mathrm{Mm}$ or ranging between 25-100 Mm with a peak at $45 \mathrm{Mm}$ ) have also been reported (Dziembowski et al. 2000; Antia et al. 2000) but it was not possible to distinguish between a thermal or a magnetic perturbation. If magnetic, estimates for the magnetic field strength ranged from $2-3 \times 10^{4} \mathrm{G}$ (Antia et al. 2000) to $6 \times 10^{4} \mathrm{G}$ (Dziembowski et al. 2000).

It is of interest to develop a model of such magnetic layers located at the base of the convection zone or in the sunspot anchoring zone. A general model is described in Sect. 2, followed by a description of the governing equations and wave solutions in Sect. 3. In Sect. 4 a dispersion relation is obtained and solved numerically. Analytical properties of the frequency shifts are elaborated in Sects. 5 and 6. An analytical approximation of these effects developed in Sect. 7 offers support for the numerics and serves to evaluate the amplitude of the shifts for perpendicular propagation. Limits of the model and possible implications of the results are discussed in Sect. 8.

\section{General model}

Diagnostics of the internal magnetic layers are obtained through a plane-parallel model, which corresponds to a description of a spherically symmetric Sun, stratified and structured radially. Let $\boldsymbol{x}, \boldsymbol{y}$ and $\boldsymbol{z}$ represent Cartesian coordinate unit vectors: $\boldsymbol{x} \boldsymbol{y}$ represent a local horizontal plane, while the depth is given in terms of the unit vector $z$, measured downwards from the solar surface (gravity $\boldsymbol{g}$ acting in the positive $z$-direction). The model includes three separate plasma layers: the magnetic layer is a slab of horizontal magnetic field lying between depths $z=z_{\mathrm{p}}$ and $z=z_{\mathrm{q}}$, and the field-free regions are hydrostatically stratified polytropes (see Fig. 1). Within the magnetic slab, the plasma is assumed to be isothermal and the horizontal field is structured in such a way as to produce a constant Alfvén speed, $v_{\mathrm{A}}$. The polytropic index of the adiabatically stratified field-free media is $m=1 /(\gamma-1)=3 / 2$, where $\gamma=5 / 3$ is the ratio of specific heats. In the polytropes, the square of the sound speed, $c_{\mathrm{s}}^{2}(z)$, has a linear profile with slope $A=g / m$, with $A=0.183 \mathrm{~m} \mathrm{~s}^{-2}$ for constant gravitational acceleration $g=274 \mathrm{~m} \mathrm{~s}^{-2}$ (taken at the solar surface $z=0$ ). The sound speed is zero at the solar surface $z=0$ and is assumed to be continuous across each magnetic interface, giving $c_{\mathrm{s}}^{2}(z)=c_{\mathrm{c}}^{2}=A z_{\mathrm{p}}$ within the slab (for $z_{\mathrm{p}} \leq z \leq z_{\mathrm{q}}$ ). At an interface between a magnetic region and a field-free region, there is a jump in the hydrostatic pressure $p_{\mathrm{o}}$, changing from $p_{\mathrm{o}}^{\mathrm{mag}}$ to $p_{\mathrm{o}}^{\text {free: }}$

$\frac{p_{\mathrm{o}}^{\mathrm{mag}}}{p_{\mathrm{o}}^{\text {free }}}=\frac{\left.\left(\rho_{\mathrm{o}} c_{\mathrm{c}}^{2}\right)\right|_{\mathrm{mag}}}{\left.\left(\rho_{\mathrm{o}} c_{\mathrm{c}}^{2}\right)\right|_{\text {free }}}=\frac{1}{1+\frac{\gamma}{2 \beta}}=\frac{\Gamma_{\mathrm{o}}}{\gamma}$,

where $\rho_{\mathrm{o}}$ is the equilibrium density, $\Gamma_{\mathrm{o}}=\gamma /(1+\gamma / 2 \beta)$ is the magnetically-modified adiabatic exponent and $\beta=c_{\mathrm{c}}^{2} / v_{\mathrm{A}}^{2}$ is the ratio between the squared sound and Alfvén speeds within the magnetic region.

One may note that the jump in hydrostatic pressure across a magnetic surface implies that if the sound speed is taken to be continuous, the field-free fluid is always denser than in the

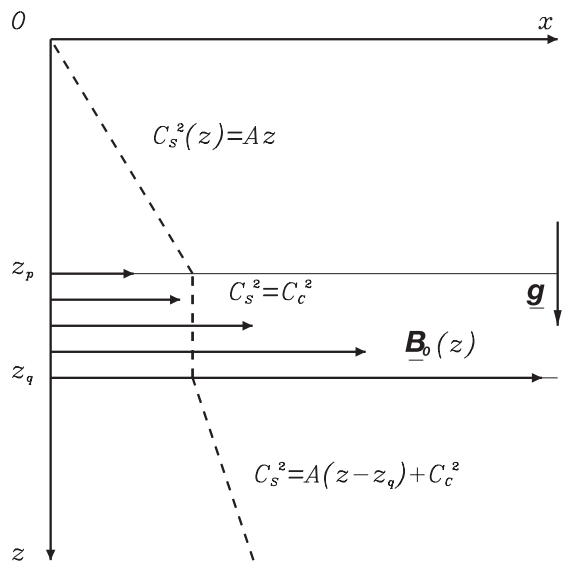

Fig. 1. Sketch of equilibrium configuration for the three-layer model and whose conditions are specified in Table 1.

Table 1. Model conditions used in the investigation of the modes of Fig. 1. The first list of parameters are related to the field-free side of the upper magnetic interface: the depth $z_{\mathrm{p}}$, the sound speed $c_{\mathrm{c}}$, the pressure scale-height $H\left(z_{\mathrm{p}}\right)$, and the hydrostatic pressure $p_{\mathrm{o}}^{\text {free }}$. The second set of parameters relate to the magnetic region: the field strength $B_{\mathrm{o}}\left(z_{\mathrm{p}}\right)$, the constant Alfvén speed $v_{\mathrm{A}}$, the ratio $\beta$ between the squared sound speed and the Alfvén speed and the density jump at the magnetic interface $\rho_{\mathrm{o}}^{\text {free }} / \rho_{\mathrm{o}}^{\mathrm{mag}}$. The third set of parameters describe properties of the model below the magnetic field: the ratio $h / z_{\mathrm{p}}$ and the depth $z_{\mathrm{q}}$ of the lower magnetic interface.

\begin{tabular}{llll}
\hline \hline \multicolumn{2}{l}{ Model parameters } & $\begin{array}{l}\text { Tachocline } \\
\text { model }\end{array}$ & $\begin{array}{l}\text { Shallow } \\
\text { field model }\end{array}$ \\
\hline$z_{\mathrm{p}}$ & $(\mathrm{km})$ & 272239 & 35036 \\
$z_{\mathrm{p}} / R_{\odot}$ & & 0.3911 & 0.0503 \\
$c_{\mathrm{c}}$ & $\left(\mathrm{km} \mathrm{s}^{-1}\right)$ & 223 & 80 \\
$H\left(z_{\mathrm{p}}\right)$ & $(\mathrm{km})$ & 108896 & 14014 \\
$H\left(z_{\mathrm{p}}\right) / R_{\odot}$ & & 0.1564 & 0.0201 \\
$p_{\mathrm{o}}^{\text {free }}$ & $(\mathrm{Pa})$ & $5.25855 \times 10^{12}$ & $5.23298 \times 10^{10}$ \\
\hline$B_{\mathrm{o}}\left(z_{\mathrm{p}}\right)$ & $(\mathrm{kG})$ & 300 & 30 \\
$v_{\mathrm{A}}$ & $(\mathrm{km} \mathrm{s})$ & 2.016 & 0.725 \\
$\beta$ & $\left(=c_{\mathrm{c}}^{2} / v_{\mathrm{A}}^{2}\right)$ & 12236.368 & 12176.864 \\
$\frac{\rho_{\text {free }}}{\rho_{\mathrm{o}}}-1$ & $\left(\times 10^{-5}\right)$ & 6.8103 & 6.8436 \\
\hline$h / R_{\odot}$ & & 0.05 & 0.05 \\
$h / z_{\mathrm{p}}$ & & 0.128 & 0.994 \\
$z_{\mathrm{q}}$ & $(\mathrm{km})$ & 307039 & 69836 \\
$z_{\mathrm{q}} / R_{\odot}$ & & 0.4411 & 0.1003 \\
\hline
\end{tabular}

magnetic region at such interface. In the general layered models that are investigated here, the field-free polytropic upper layer overlies a magnetic layer at the interface $z=z_{\mathrm{p}}$. At this interface, the jump in density (1) yields a magnetic buoyancy force of $\left(\rho_{\mathrm{o}}^{\text {free }}-\rho_{\mathrm{o}}^{\mathrm{mag}}\right) g$ per unit volume. Consequently the "topheavy" equilibrium state may be unstable. This effect causes magnetic field to erupt through the convection zone and into the upper layers. We do not explore this effect here.

Two situations are considered: those of a tachocline model and those of a shallow field model. The parameters for these models are chosen to best represent the two magnetic field configurations; see Table 1. The tachocline model includes a magnetic layer assumed to reside at the base of the convection zone, 
with a field strength of $300 \mathrm{kG}$ at $z=z_{\mathrm{p}}$; the shallow field model includes the sunspot's anchoring zone, with a field strength of $30 \mathrm{kG}$ at $z=z_{\mathrm{p}}$. The value for the sound speed at the base of the convection zone is determined, from helioseismic inversion techniques, by Christensen-Dalsgaard et al. (1991), who give $c_{\mathrm{c}}=223 \pm 2 \mathrm{~km} \mathrm{~s}^{-1}$. The depth $z_{\mathrm{p}}$ is deduced through the relation $c_{\mathrm{c}}^{2}=A z_{\mathrm{p}}$. For the shallow field model, the sound speed $c_{\mathrm{c}}=80 \mathrm{~km} \mathrm{~s}^{-1}$ was chosen so as to obtain $z_{\mathrm{p}}$ and $z_{\mathrm{q}}$ in the range identified to be the seat of a significant perturbation (Dziembowski et al. 2000; Antia et al. 2000). In both models, the thickness of the magnetic layer is taken to be $0.05 R_{\odot}$. For the shallow field model, this corresponds to a magnetic layer extending from a depth of $35 \mathrm{Mm}$ down to $70 \mathrm{Mm}$. It may be noted that this shallow layer extends up to where the torsional oscillations appear to be significant (see, e.g., Hathaway et al. 1996; Schou et al. 1998; Howe et al. 2000; Antia \& Basu 2000). The hydrodynamic pressures are chosen to match the sound speeds in the solar model of Guenther et al. (1992), thus setting conditions as close as possible to those in the solar interior. The value of the Alfvén speed $v_{\mathrm{A}}$ is obtained from the magnetic field strength $B_{\mathrm{o}}$ at depth $z_{\mathrm{p}}$ through

$v_{\mathrm{A}}=\left(\frac{B_{\mathrm{o}}^{2}\left(z_{\mathrm{p}}\right)}{\mu \rho_{\mathrm{o}}^{\mathrm{mag}}\left(z_{\mathrm{p}}\right)}\right)^{1 / 2}$,

where $\mu\left(=4 \pi \times 10^{-7} \mathrm{H} \mathrm{m}^{-1}\right)$ is the magnetic permeability.

\section{Wave equations and solutions}

In the Cartesian system $(x, y, z)$, the applied horizontal magnetic field is taken to be directed along the $x$-axis. The perturbations of the ideal linearised MHD equations (for constant gravitational acceleration $g$ and without background flow) are Fourier analysed proportionally to $\exp \mathrm{i}\left(\omega t-k_{x} x-k_{y} y\right)$, where $\omega$ is the angular frequency of a mode of horizontal wavenumber $k_{\mathrm{h}}$ with $\boldsymbol{k}_{\mathrm{h}}=\left(k_{x}, k_{y}, 0\right)$. The horizontal wavenumber $k_{\mathrm{h}}$ is related to the degree $l$ through $k_{\mathrm{h}}=L / R_{\odot}$, for $L=\sqrt{l(l+1)}$ and solar radius $R_{\odot}$.

\subsection{Fundamental wave equation}

Wave propagation can be described in terms of the vertical velocity perturbation $V_{z}$ and the Lagrangian rate of change in total pressure perturbation, $\mathcal{P}_{\mathrm{T}}$.

The governing differential equation satisfied by the vertical velocity component $V_{z}$ may be written in the form

$\frac{1}{\rho_{\mathrm{o}}(z)} \frac{\mathrm{d}}{\mathrm{d} z}\left[A_{1}(z) \rho_{\mathrm{o}}(z) \frac{\mathrm{d} V_{z}}{\mathrm{~d} z}\right]+A_{2}(z) V_{z}=0$,

where the coefficients $A_{1}$ and $A_{2}$ are given by

$$
\begin{aligned}
A_{1}(z)= & \frac{\left(c_{\mathrm{s}}^{2}+v_{\mathrm{A}}^{2}\right)\left(\omega^{2}-k_{x}^{2} c_{\mathrm{T}}^{2}\right)}{\omega^{2}-k_{x}^{2} c_{\mathrm{s}}^{2}-k_{y}^{2} c_{\perp}^{2}}, \\
A_{2}(z)= & \left(\omega^{2}-k_{x}^{2} v_{\mathrm{A}}^{2}\right)-\frac{g^{2}\left(k_{x}^{2}+k_{y}^{2}\right)}{\left(\omega^{2}-k_{x}^{2} c_{\mathrm{s}}^{2}-k_{y}^{2} c_{\perp}^{2}\right)} \\
& +\frac{g}{\rho_{\mathrm{o}}} \frac{\mathrm{d}}{\mathrm{d} z}\left[\rho_{\mathrm{o}}(z) \frac{k_{x}^{2} c_{\mathrm{s}}^{2}+k_{y}^{2} c_{\perp}^{2}}{\omega^{2}-k_{x}^{2} c_{\mathrm{s}}^{2}-k_{y}^{2} c_{\perp}^{2}}\right] .
\end{aligned}
$$

Here $c_{\mathrm{T}}$ is the magnetoacoustic cusp speed, expressed through

$c_{\mathrm{T}}^{2}=\frac{c_{\mathrm{s}}^{2} v_{\mathrm{A}}^{2}}{v_{\mathrm{A}}^{2}+c_{\mathrm{s}}^{2}}$

$c_{\perp}$ is independent of $k_{y}$ and defined through

$c_{\perp}^{2}=\frac{\omega^{2}}{\omega^{2}-k_{x}^{2} v_{\mathrm{A}}^{2}}\left(c_{\mathrm{s}}^{2}+v_{\mathrm{A}}^{2}-\frac{k_{x}^{2}}{\omega^{2}} c_{\mathrm{s}}^{2} v_{\mathrm{A}}^{2}\right)$.

A detailed derivation of Eq. (3) is given in Goedbloed (1971), Nye \& Thomas (1976) and Foullon (2002a). Here and in Foullon (2002a) we emphasise the use of $c_{\perp}(z)$. In the fieldfree limit, $c_{\perp} \rightarrow c_{\mathrm{s}}$. The pressure perturbations are given in Appendix A.

\subsection{Polytropic layers}

The governing equation for $V_{z}$ in a field-free plasma follows from Eq. (3), taking $v_{\mathrm{A}}$ and $c_{\mathrm{T}}$ to be zero. But this equation possesses a complex structure, even in the field-free limit. It proves more convenient to describe the motion in terms of the compressibility $\Delta$ by way of Lamb's equation (Lamb 1932) rewritten in the standard form (see, e.g., Deubner \& Gough 1984)

$\frac{\mathrm{d}^{2} Q}{\mathrm{~d} z^{2}}+K^{2}(z) Q=0$

where $Q(z)=\sqrt{\rho_{\mathrm{o}}(z)} c_{\mathrm{s}}^{2}(z) \Delta$ and

$K^{2}(z)=\frac{\omega^{2}-\omega_{\mathrm{a}}^{2}}{c_{\mathrm{s}}^{2}(z)}+k_{\mathrm{h}}^{2}\left(\frac{\omega_{\mathrm{g}}^{2}}{\omega^{2}}-1\right)$.

Here the buoyancy frequency $\omega_{\mathrm{g}}$ is given by

$\omega_{\mathrm{g}}^{2}=\frac{g}{H_{\rho}}-\frac{g^{2}}{c_{\mathrm{s}}^{2}(z)}=\frac{g}{c_{\mathrm{s}}^{2}(z)}\left[(\gamma-1) g-\left[c_{\mathrm{s}}^{2}(z)\right]^{\prime}\right]$

and $\omega_{\mathrm{a}}^{2}$ is the squared acoustic cut-off frequency (Lamb 1909, 1932) defined through

$\omega_{\mathrm{a}}^{2}=\omega_{\mathrm{o}}^{2}\left(1+2 H_{\rho}^{\prime}\right), \quad \omega_{\mathrm{o}}^{2}=\frac{c_{\mathrm{s}}^{2}(z)}{4 H_{\rho}^{2}}$.

Lamb's equation applied to a polytrope (Lamb 1932) has solution

$\Delta=\mathrm{e}^{-\frac{Z}{2}}\left[\alpha_{1} M(-\Upsilon, m+2, Z)+\beta_{1} U(-\Upsilon, m+2, Z)\right]$,

where $\alpha_{1}$ and $\beta_{1}$ are arbitrary constants and $2 \Upsilon=m \Omega^{2}-(m+$ 2) where $\Omega$ is the normalised eigenfrequency such that $\Omega^{2}=$ $\omega^{2} /\left(g k_{\mathrm{h}}\right)$; the functions $M$ and $U$ are respectively the Kummer and Tricomi confluent hypergeometric functions (Slater 1965), evaluated at $Z=2 k_{\mathrm{h}} z \geq 0$.

The case of a vanishing sound speed at the surface leads to the selection of the Kummer $M$ function. In a simple field-free polytrope model (Spiegel \& Unno 1962; Christensen-Dalsgaard 1980), which does not contain a magnetic slab, the condition of boundedness as $z \rightarrow \infty$ requires the $M$ functions to terminate in the form of generalised Laguerre polynomials, and the parameter $\Upsilon$ has to be a non-negative integer. The solutions are the $p$-modes, given by

$\Omega^{2}=\Omega_{n}^{2} \equiv 1+\frac{2 n}{m}$

for integer $n \geq 0$. 
Table 2. Elements of the magneto-atmospheric wave equation in certain limiting cases, corresponding to the field-free limit $\left(v_{\mathrm{A}}=0\right)$, parallel propagation $\left(k_{y}=0\right)$ and perpendicular propagation $\left(k_{x}=0\right)$.

\begin{tabular}{cccc}
\hline \hline & $v_{\mathrm{A}}=0$ & $k_{y}=0$ & $k_{x}=0$ \\
\hline$c_{\perp}^{2}$ & $c_{\mathrm{c}}^{2}$ & $c_{\perp}^{2}$ & $c_{\mathrm{c}}^{2}+v_{\mathrm{A}}^{2}$ \\
$H_{\mathrm{o}}$ & $\frac{c_{\mathrm{c}}}{\gamma g}=H$ & $H_{\mathrm{o}}$ & $H_{\mathrm{o}}$ \\
$\varpi^{2}$ & $\omega^{2}$ & $\varpi^{2}$ & $\frac{\left(c_{\mathrm{c}}^{2}+v_{\mathrm{A}}^{2}\right)}{c_{\mathrm{c}}^{2}} \omega^{2}$ \\
$\tilde{\omega}_{\mathrm{a}}^{2}$ & $\frac{c_{\mathrm{c}}^{2}}{4 H^{2}}=\omega_{\mathrm{a}}^{2}$ & $\tilde{\omega}_{\mathrm{a}}^{2}$ & $\frac{c_{\mathrm{c}}^{2}+v_{\mathrm{A}}^{2}}{4 H_{\mathrm{o}}^{2}}=\tilde{\omega}_{\mathrm{a} \perp}^{2}$ \\
$\tilde{\omega}_{\mathrm{g}}^{2}$ & $\frac{g}{H}-\frac{g^{2}}{c_{\mathrm{c}}^{2}}=\omega_{\mathrm{g}}^{2}$ & $\frac{g}{H_{\mathrm{o}}}-\frac{g^{2}}{c_{\mathrm{c}}^{2}}=\tilde{\omega}_{\mathrm{g} \|}^{2}$ & $\frac{g}{H_{\mathrm{o}}}-\frac{g^{2}}{c_{\mathrm{c}}^{2}+v_{\mathrm{A}}^{2}}=\tilde{\omega}_{\mathrm{g} \perp}^{2}$ \\
$\kappa^{2}$ & $\frac{\omega^{2}-\omega_{\mathrm{a}}^{2}}{c_{\mathrm{c}}^{2}}+k_{\mathrm{h}}^{2} \frac{\omega_{\mathrm{g}}^{2}-\omega^{2}}{\omega^{2}}$ & $\frac{\frac{\omega^{4}}{\omega^{2}}-\omega_{\mathrm{ao}}^{2}}{c_{\mathrm{c}}^{2}}+k_{x}^{2} \frac{\tilde{\omega}_{\mathrm{g} \|}^{2}-\varpi^{2}}{\omega^{2}}$ & $\frac{\omega^{2}-\tilde{\omega}_{\mathrm{a} \perp}^{2}}{c_{\mathrm{c}}^{2}+v_{\mathrm{A}}^{2}}+k_{y}^{2} \frac{\tilde{\omega}_{\mathrm{g} \perp}^{2}-\omega^{2}}{\omega^{2}}$ \\
\hline
\end{tabular}

\subsection{Isothermal layers}

It is instructive to simplify the fundamental Eq. (3) when the sound and Alfvén speeds are constant, viz.

$\frac{\mathrm{d}^{2} V_{z}}{\mathrm{~d} z^{2}}+\frac{1}{H_{\mathrm{o}}} \frac{\mathrm{d} V_{z}}{\mathrm{~d} z}+A_{\mathrm{o}} V_{z}=0$

where

$$
\begin{aligned}
A_{\mathrm{o}}=\frac{A_{2}}{A_{1}}= & \frac{\omega^{2}-k_{x}^{2} c_{\mathrm{c}}^{2}-k_{y}^{2} c_{\perp}^{2}}{c_{\perp}^{2}} \\
& +\frac{k_{x}^{2} c_{\mathrm{c}}^{2}+k_{y}^{2} c_{\perp}^{2}}{c_{\perp}^{2}\left(\omega^{2}-k_{x}^{2} v_{\mathrm{A}}^{2}\right)}\left[\frac{g}{H_{\mathrm{o}}}-\frac{g^{2}\left(k_{x}^{2}+k_{y}^{2}\right)}{k_{x}^{2} c_{\mathrm{c}}^{2}+k_{y}^{2} c_{\perp}^{2}}\right]
\end{aligned}
$$

and $H_{\mathrm{o}}$ is the density scale-height, viz.

$H_{\mathrm{o}}=\rho_{\mathrm{o}}\left(\frac{\mathrm{d} \rho_{\mathrm{o}}}{\mathrm{d} z}\right)^{-1}=\frac{c_{\mathrm{c}}^{2}}{\Gamma_{\mathrm{o}} g}=\frac{c_{\mathrm{c}}^{2}}{\gamma g}\left(1+\frac{\gamma}{2 \beta}\right)$.

With the transformation

$W(z)=V_{z} \mathrm{e}^{\frac{z-z \mathrm{p}}{2 H_{0}}}$,

where $z_{\mathrm{p}}$ is an arbitrary reference depth in the stratified medium, Eq. (14) assumes the standard form

$\frac{\mathrm{d}^{2} W(z)}{\mathrm{d} z^{2}}+\kappa^{2} W(z)=0$

with $\kappa^{2}$ a constant.

Table 2 gives a summary of the elements of the magnetoatmospheric wave equation for an isothermal field-free limit, as well as for the limiting cases of parallel and perpendicular propagation, noting the following identifications: $\tilde{\omega}_{\mathrm{a}}$ is the magnetically modified acoustic cut-off for an isothermal medium, defined through

$\tilde{\omega}_{\mathrm{a}}^{2}=\frac{c_{\perp}^{2}}{4 H_{\mathrm{o}}^{2}}=\omega_{\mathrm{ao}}^{2} \frac{\varpi^{2}}{\omega^{2}-k_{x}^{2} v_{\mathrm{A}}^{2}}$,

with

$\omega_{\mathrm{ao}}^{2}=\frac{c_{\mathrm{c}}^{2}}{4 H_{\mathrm{o}}^{2}}=\frac{g^{2} \Gamma_{\mathrm{o}}^{2}}{4 c_{\mathrm{c}}^{2}} ;$ $\tilde{\omega}_{\mathrm{g}}$ is the magnetically modified buoyancy frequency given by

$\tilde{\omega}_{\mathrm{g}}^{2}=\frac{g}{H_{\mathrm{o}}}-\frac{g^{2}\left(k_{x}^{2}+k_{y}^{2}\right)}{k_{x}^{2} c_{\mathrm{c}}^{2}+k_{y}^{2} c_{\perp}^{2}}$

$\varpi^{2}$ is the squared magnetically modified frequency:

$\varpi^{2}=\frac{c_{\mathrm{c}}^{2}+v_{\mathrm{A}}^{2}}{c_{\mathrm{c}}^{2}}\left(\omega^{2}-k_{x}^{2} c_{\mathrm{T}}^{2}\right)=\omega^{2}\left(1+\frac{1}{\beta}\right)-k_{x}^{2} v_{\mathrm{A}}^{2}$.

The vertical velocity amplitude $V_{z}$ is a solution of the wave equation in the magnetic region (cf. Campbell \& Roberts 1989, when $k_{y}=0$ )

$V_{z}=D_{1} \mathrm{e}^{\Lambda^{+} z}+D_{2} \mathrm{e}^{\Lambda^{-} z}$,

with

$\Lambda^{ \pm}=-1 /\left(2 H_{\mathrm{o}}\right) \pm \kappa$

the scale $\kappa$ is real for surface modes and imaginary for oscillatory modes in the layer.

\section{Dispersion relation}

At an interface $z=z_{i}, i \in\{\mathrm{p}, \mathrm{q}\}$, the wave dynamics is coupled by the condition

$\left.\frac{V_{z}}{\mathcal{P}_{\mathrm{T}}}\right|_{\text {mag }}=\left.\frac{V_{z}}{\mathcal{P}_{\mathrm{T}}}\right|_{\text {free }} ;$

the field-free part is

$\left.\frac{V_{z}}{\mathcal{P}_{\mathrm{T}}}\right|_{\text {free }}=\left.\frac{V_{z}}{-\rho_{\mathrm{o}} c_{\mathrm{c}}^{2} \Delta}\right|_{\text {free }}$,

while the magnetic part is (see Eq. (A.16))

$\left.\frac{V_{z}}{\mathcal{P}_{\mathrm{T}}}\right|_{\mathrm{mag}}=\left.\frac{V_{z}}{-\rho_{\mathrm{o}} c_{\mathrm{c}}^{2}\left(\Delta+\frac{1}{\beta} \Delta_{\perp}\right)}\right|_{\mathrm{mag}}$.

Here $\Delta$ is the compressibility of the plasma and $\Delta_{\perp}$ gathers the componenents of compressibility perpendicular to the magnetic field lines, viz.

$\Delta_{\perp}=\frac{\partial V_{y}}{\partial y}+\frac{\partial V_{z}}{\partial z}=\Delta-\frac{\partial V_{x}}{\partial x}$.

Equating expressions (26) and (27), and substituting in the jump in hydrostatic pressure (1), the coupling relation at the magnetic interface (25) is reduced to

$\left.\mathcal{D}\right|_{\text {free }}=\left.\mathcal{D}_{\mathrm{o}}\right|_{\mathrm{mag}}$

where $\mathcal{D}\left(\Omega^{2}, k_{\mathrm{h}}, c_{\mathrm{s}}^{2}(z)\right)$, the non-magnetic part, is the ratio $V_{z} / \Delta$ for a field-free medium, viz.

$\mathcal{D}=\frac{1}{\Omega^{4}-1}\left[\frac{c_{\mathrm{s}}^{2}}{g}-\frac{\gamma \Omega^{2}}{k_{\mathrm{h}}}-\frac{c_{\mathrm{s}}^{2} \Omega^{2}}{g k_{\mathrm{h}}} \frac{1}{\Delta} \frac{\mathrm{d} \Delta}{\mathrm{d} z}\right]$,

and $\mathcal{D}_{\mathrm{o}}\left(\Omega^{2}, k_{x}, k_{y}, c_{\mathrm{c}}^{2}, \beta\right)$ is the magnetic part

$\mathcal{D}_{\mathrm{o}}=\frac{Q}{P \frac{1}{V_{z}} \frac{\mathrm{d} V_{z}}{\mathrm{~d} z}+R}$, 
where

$P=\left(1+\frac{1}{\beta}\right)\left(\Omega^{2}-\frac{k_{x}^{2} c_{\mathrm{T}}^{2}}{g k_{\mathrm{h}}}\right), \quad Q=\frac{\gamma}{\Gamma_{\mathrm{o}}}\left(\Omega^{2}-\frac{k_{x}^{2} c_{\mathrm{c}}^{2}+k_{y}^{2} c_{\perp}^{2}}{g k_{\mathrm{h}}}\right)$,

$R=\frac{k_{x}^{2} c_{\mathrm{c}}^{2}+k_{y}^{2} c_{\perp}^{2}}{c_{\mathrm{c}}^{2}}$

To obtain the dispersion relation, we develop the coupling condition (29) at an interface $z=z_{i}$ :

$\mathcal{D}\left(R+P \frac{1}{V_{z}} \frac{\mathrm{d} V_{z}}{\mathrm{~d} z}\right)-Q=0$.

With $\frac{1}{V_{z}} \frac{\mathrm{d} V_{z}}{\mathrm{~d} z}$ evaluated at $z=z_{i}$, viz.

$\frac{1}{V_{z}} \frac{\mathrm{d} V_{z}}{\mathrm{~d} z}=\frac{D_{1} \Lambda^{+} \mathrm{e}^{\Lambda^{+} z_{i}}+D_{2} \Lambda^{-} \mathrm{e}^{\Lambda^{-} z_{i}}}{D_{1} \mathrm{e}^{\Lambda^{+} z_{i}}+D_{2} \mathrm{e}^{\Lambda^{-} z_{i}}}$,

the requirement (33) yields

$D_{1}\left[\left(R+P \Lambda^{+}\right) \mathcal{D}-Q\right] \mathrm{e}^{\Lambda^{+} z_{i}}+D_{2}\left[\left(R+P \Lambda^{-}\right) \mathcal{D}-Q\right] \mathrm{e}^{\Lambda^{-} z_{i}}=0$.

Taking $z_{i}=z_{\mathrm{p}}$ and $z_{i}=z_{\mathrm{q}}$ in turn leads to the dispersion relation

$\mathcal{E}_{\mathrm{p}}^{-} \mathcal{E}_{\mathrm{q}}^{+} \mathrm{e}^{2 \kappa\left(z_{\mathrm{q}}-z_{\mathrm{p}}\right)}-\mathcal{E}_{\mathrm{p}}^{+} \mathcal{E}_{\mathrm{q}}^{-}=0$

where

$\mathcal{E}_{\mathrm{p}}^{ \pm}=\left(R+P \Lambda^{ \pm}\right) \mathcal{D}_{\mathrm{p}}-Q, \quad \mathcal{E}_{\mathrm{q}}^{ \pm}=\left(R+P \Lambda^{ \pm}\right) \mathcal{D}_{\mathrm{q}}-Q$.

The dispersion relation (36) may be compared to the transmittance of a four-terminal network, where the magnetic slab corresponds to an electrical system or black box with two input and two output terminals. The respective input and output impedances are given by the field-free parts $\mathcal{D}_{\mathrm{p}}$ and $\mathcal{D}_{\mathrm{q}}$, obtained by considering the general expression (30) at $z=z_{\mathrm{p}}$ and $z=z_{\mathrm{q}}$.

The requirement of a free-moving surface leads to a study of the isolated effect of a buried magnetic field on $p$-modes. This is readily obtained for the case of a vanishing sound speed at the surface, leading to the selection of the Kummer $M$ function; then

$\mathcal{D}_{\mathrm{p}}=\frac{z_{\mathrm{p}}}{m\left(\Omega^{4}-1\right)}\left[1-\frac{(m+1)}{k_{\mathrm{h}} z_{\mathrm{p}}} \Omega^{2}+\Omega^{2}-2 \Omega^{2} \frac{M^{\prime}}{M}\right]$.

For a polytrope below $z_{\mathrm{q}}$, the requirement of vanishing kinetic energy density at $z \rightarrow \infty$ is satisfied by selecting the Tricomi $U$ function; then

$\mathcal{D}_{\mathrm{q}}=\frac{z_{\mathrm{p}}}{m\left(\Omega^{4}-1\right)}\left[1-\frac{(m+1)}{k_{\mathrm{h}} z_{\mathrm{p}}} \Omega^{2}+\Omega^{2}-2 \Omega^{2} \frac{U^{\prime}}{U}\right]$.

In Eqs. (38) and (39), $M, U, M^{\prime}$ and $U^{\prime}$ are evaluated at $Z=$ $2 k_{\mathrm{h}} z_{\mathrm{p}}$, and we have written

$$
\begin{aligned}
M=M(-\Upsilon, m+2, Z), & U=U(-\Upsilon, m+2, Z), \\
M^{\prime}=M^{\prime}(-\Upsilon, m+2, Z), & U^{\prime}=U^{\prime}(-\Upsilon, m+2, Z),
\end{aligned}
$$

where the prime denotes the derivative of $M$ or $U$ with respect to $Z$.

Eigenfunctions in the respective layers are derived in Appendix B.

\section{General analytical properties}

Some properties of the frequency shifts can be explained analytically. For example, an increase in magnetic field strength raises the frequency shift proportionally to the square of the field strength: a magnetic slab of field strength ten times larger than the original yields shifts of amplitude hundred times bigger. This property was first obtained analytically for fibril magnetic fields (Bogdan \& Zweibel 1985; Zweibel \& Bogdan 1986), and for propagation parallel to an horizontal magnetic layer (Roberts \& Campbell 1986; Campbell 1987; Daniell 1998). In Zweibel \& Bogdan (1986), the dependence on the magnetic field strength was also expressed for a polytropic slab.

The property can be understood as follows. As the frequency relates to the local sonic speed $c(\omega=c / k)$, a change in frequency indicates a change in local sonic speed. For a Lagrangian change in pressure $\delta p$ in a medium of density $\rho_{\mathrm{o}}$ and compressibility $\Delta$, the local sonic speed $c$ is given by

$c^{2}=\frac{\delta p}{-\rho_{\mathrm{o}} \Delta}$.

In the presence of a horizontal magnetic field, the wave experiences an effective magnetosonic speed $c$ given by (see Eq. (A.16))

$c^{2}=c_{\mathrm{s}}^{2}\left[1+\frac{v_{\mathrm{A}}^{2}}{c_{\mathrm{s}}^{2}} \frac{\Delta_{\perp}}{\Delta}\right]$,

indicating that the resulting change in frequency due to the presence of a magnetic field is proportional to $v_{\mathrm{A}}^{2} / c_{\mathrm{s}}^{2}$ and so broadly proportional to the square of the magnetic field strength.

However, one cannot argue that the change in frequency is generally inversely proportional to the plasma $\beta\left(=c_{\mathrm{s}}^{2} / v_{\mathrm{A}}^{2}\right)$ : Eq. (43) shows that the effect increases with the normalised component of compressibility perpendicular to the magnetic field lines, $\Delta_{\perp} / \Delta$. Gough \& Thompson (1990) obtained a similar result through perturbation theory. They found (see their Eq. (4.10)) that the effect increases instead with $\sin ^{2} \psi$, where $\psi$ is the angle between the magnetic field and the direction of propagation of the wave. Hence the magnitude of the perturbation depends on the direction of propagation of the wave.

Zweibel \& Bogdan (1986) showed that for a submerged layer of horizontal flux tubes, modes which turn within the layer are more strongly affected. At the turning depth of the mode, the propagation is parallel to the horizontal direction, but not necessarily to the magnetic field lines. Zweibel and Bogdan also showed clearly that the effect is larger for modes propagating perpendicular to the symmetry axis of the tubes at the turning depth (i.e. $k_{y} \rightarrow \infty$ ), rather than parallel propagating modes $\left(k_{y}=0\right)$.

The dependence of $\Delta_{\perp} / \Delta$ on $\beta$ must be studied more carefully. The ratio $\Delta_{\perp} / \Delta$ is given by

$\frac{\Delta_{\perp}}{\Delta}=1-\frac{k_{x}^{2} c_{\mathrm{s}}^{2}}{\omega^{2}}-\frac{g k_{x}^{2}}{\omega^{2}} \frac{V_{z}}{\Delta}$.

If $k_{x}=0$, the mode propagates perpendicular to the magnetic field lines and $\Delta_{\perp}=\Delta$, so the characteristic speed of this mode (see Eq. (43)) is the fast magnetoacoustic speed $\left(c_{\mathrm{s}}^{2}+v_{\mathrm{A}}^{2}\right)^{\frac{1}{2}}$. 
Thus, for propagation perpendicular to magnetic field lines, the effective fast magnetoacoustic speed serves to increase the frequency of the mode.

Equation (44) supports the idea that the parallel propagating modes $\left(k_{x} \neq 0\right)$ would be less affected by the magnetic field. In the limit of zero gravity, this is clear, viz.

$$
\frac{\Delta_{\perp}}{\Delta}=1-\frac{k_{x}^{2} c_{\mathrm{s}}^{2}}{\omega^{2}}, \quad(g=0)
$$

but in the general case, unless $V_{z}$ and $\Delta$ have the same sign, the effect requires further study.

Gravity brings about changes that depend on $k_{y}$. The compressibility $\Delta$ and the vertical velocity $V_{z}$ are related through

$$
\left(1-\frac{k_{x}^{2} c_{\mathrm{s}}^{2}+k_{y}^{2} c_{\perp}^{2}}{\omega^{2}}\right) \Delta=\frac{g\left(k_{x}^{2}+k_{y}^{2}\right)}{\omega^{2}} V_{z}+\frac{\mathrm{d} V_{z}}{\mathrm{~d} z} .
$$

The correction to Eq. (45) due to gravity is obtained by rewriting the right-hand side of Eq. (44) in replacing $\Delta$ from Eq. (46), viz.

$$
\frac{\Delta_{\perp}}{\Delta}=\left(1-\frac{k_{x}^{2} c_{\mathrm{s}}^{2}}{\omega^{2}}\right) \times\left(1-\frac{1-\frac{k_{y}^{2} c_{\perp}^{2}}{\left(\omega^{2}-k_{x}^{2} c_{\mathrm{s}}^{2}\right)}}{1+\frac{k_{y}^{2}}{k_{x}^{2}}+\frac{\omega^{2}}{g k_{x}^{2}} \frac{1}{V_{z}} \frac{\mathrm{d} V_{z}}{\mathrm{~d} z}}\right)
$$

This expression indicates that the correction due to gravity involves the ratio between the gradient in vertical velocity amplitude with depth to the amplitude itself. The effect due to this term is amplified for low parallel wavenumbers $\left(k_{x} \rightarrow 0\right)$.

Furthermore, the effect of the buried magnetic layer is related to the internal and external environment of the layer, as can be seen through the ratio $\frac{1}{V_{z}} \frac{\mathrm{d} V_{z}}{\mathrm{~d} z}$ at depth $z$ in the layer (see Eq. (B.12)):

$$
\frac{1}{V_{z}} \frac{\mathrm{d} V_{z}}{\mathrm{~d} z}=-\frac{1}{2 H_{\mathrm{o}}}+\kappa \frac{\mathrm{e}^{2 \kappa\left(z-z_{\mathrm{p}}\right)}+\frac{\mathcal{E}_{\mathrm{p}}^{+}}{\mathcal{E}_{\mathrm{p}}^{-}}}{\mathrm{e}^{2 \kappa\left(z-z_{\mathrm{p}}\right)}-\frac{\mathcal{E}_{\mathrm{p}}^{+}}{\mathcal{E}_{\mathrm{p}}^{-}}}
$$

For a mode with long wavelength, viz. $\kappa \ll 1$, the ratio $\frac{\mathcal{E}_{\mathrm{p}}^{+}}{\mathcal{E}_{\mathrm{p}}^{-}} \approx 1$. For modes of wavelength much longer than the thickness of the layer, i.e. $\kappa h \ll 1$, the exponential term can be expanded $\mathrm{e}^{2 \kappa h} \approx 1+2 \kappa h$, and the expression (48) approximated by

$\frac{1}{V_{z}} \frac{\mathrm{d} V_{z}}{\mathrm{~d} z} \sim \frac{1}{h}-\frac{1}{2 H_{\mathrm{o}}}$.

If $h \sim 2 H_{0}$, the vertical velocity amplitude stays constant. If $h \gg H_{\mathrm{o}}$, then the vertical velocity amplitude decreases. For a thin layer with $h \ll H_{\mathrm{o}}$, the vertical velocity amplitude increases on the spatial scale of the layer.

The pressure or density scale-height in the isothermal layer is increased by the presence of the magnetic field, which adds support to the plasma through the finite plasma $\beta$ (see Eq. (16)). Hence, through the background equilibrium the magnetic field helps to increase the vertical gradient of the vertical velocity. As regards Eq. (47), this globally illustrates a rather complex dependence of $\Delta_{\perp} / \Delta$ on $\beta$, which may be avoided for large plasma $\beta$.

The wave equation in the magnetic layer was greatly simplified by assuming the sound and Alfvén speeds to be constant.
This assumption is justified for a thin magnetic layer, $h \ll H_{\mathrm{o}}$. Note that the ratio $\beta=c_{\mathrm{c}}^{2} / v_{\mathrm{A}}^{2}$ is very large. In these conditions, because $H_{\mathrm{o}} \gtrsim H\left(z_{\mathrm{p}}\right)$ (where $H(z)=c_{\mathrm{s}}^{2}(z) /(\gamma g)$; see Eq. (16)), only the tachocline model meets the assumption of a thin magnetic layer, as can be seen in Table 1 .

The consideration of equations in the plane-wave approximation is not strictly valid for low-degree modes. However, the plane-wave approximation may be considered valid locally if there is no significant variation along a wave front over a distance comparable to the local wavelength $\lambda$. In the isothermal layer, this condition corresponds to $\lambda \ll H_{\mathrm{o}}$, where $H_{\mathrm{o}}$ is both the density and pressure scale-heights. The local wavelength of a $p$-mode in the isothermal layer is roughly $\lambda \simeq \frac{c_{c}}{v}$. With a frequency $v \simeq 3.3 \mathrm{mHz}$ and $c_{\mathrm{c}}=223 \mathrm{~km} \mathrm{~s}^{-1}$ at the base of the convection zone, $\lambda \simeq 0.1 R_{\odot}$. For the tachocline model, this is a reasonable approximation since $H_{\mathrm{o}} \simeq 0.15 R_{\odot}$. But for a shallow field model, with $c_{\mathrm{c}}=80 \mathrm{~km} \mathrm{~s}^{-1}, \lambda \simeq 0.03 R_{\odot}>H_{\mathrm{o}} \simeq 0.02 R_{\odot}$ (see Table 1). This should be bear in mind when interpreting the resulting frequency shifts.

\section{Frequency shifts for parallel propagation}

The confluent hypergeometric functions are computed numerically and the dispersion relation (36) is solved via a root finding program. We consider the case of parallel propagation $\left(k_{y}=0\right)$ and examine the two model conditions described in Table 1. Results for the tachocline model are given in the form of normalised and dimensional diagnostic diagrams in Fig. 2.

The dispersion relation considered is function of the dimensionless combination $k_{x} z_{\mathrm{p}}$ and the dimensionless ratio $h / z_{\mathrm{p}}$. Thus increasing $l$, i.e. increasing $k_{x}$, amounts to increasing $z_{\mathrm{p}}$ and $h$ in the dispersion relation. In other words, the investigation of the dispersion relation through a range in degree $l$ is equivalent to increasing $z_{\mathrm{p}}$ and $h$ at fixed $l$. In the normalised diagnostic diagram, the roots (in full circles) of the dispersion relation converge for increasing $l$ to discrete horizontal ridges $\Omega_{n}^{2}$ in broken lines, given by Eq. (13) and such that $\Omega_{n}^{2}=1+\frac{2 n}{m}$ for radial order $n$. Similarly, in the dimensional diagnostic diagram (cyclic frequency $v$ against degree $l$ ), these roots are aligned on the ridges of $p$-modes for high degree $l$ and deflected from them as the degree decreases. That the roots collapse on the ridges of $p$-modes for high degree $l$ or when $k_{x} \rightarrow \infty$ confirms the results (13) on the basis of a simple polytrope model where $z_{\mathrm{p}} \rightarrow \infty$. Thus, in the absence of a layer, the model spectrum recovers the field-free one.

The isothermal layer may support (oscillatory) body waves $\left(\kappa^{2}>0\right)$, which exist in the shaded domains, shown in Fig. 2, separated by two cut-offs of high and low-frequency. The two cut-offs, given by the equation $\kappa^{2}=0$ and denoted $\omega_{+}^{2}$ and $\omega_{-}^{2}$, correspond to the "plus" and "minus" modes with no vertical propagation discussed in Nye and Thomas (1974), where the wave equation is Fourier analysed in the vertical direction. $g$-modes, which are allowed by the presence of the isothermal layer, propagate in the oscillatory domain below the minus cutoff, $\omega_{-}$. For $k_{x} \rightarrow \infty$ and a weak magnetic field, the high frequency separation $\omega_{+}$approaches the line $\omega=k_{x} c_{\mathrm{c}}$ (the Lamb mode in the field-free case), while the low frequency separation $\omega_{-}$approaches the line $\omega=k_{x} v_{\mathrm{A}}$. 

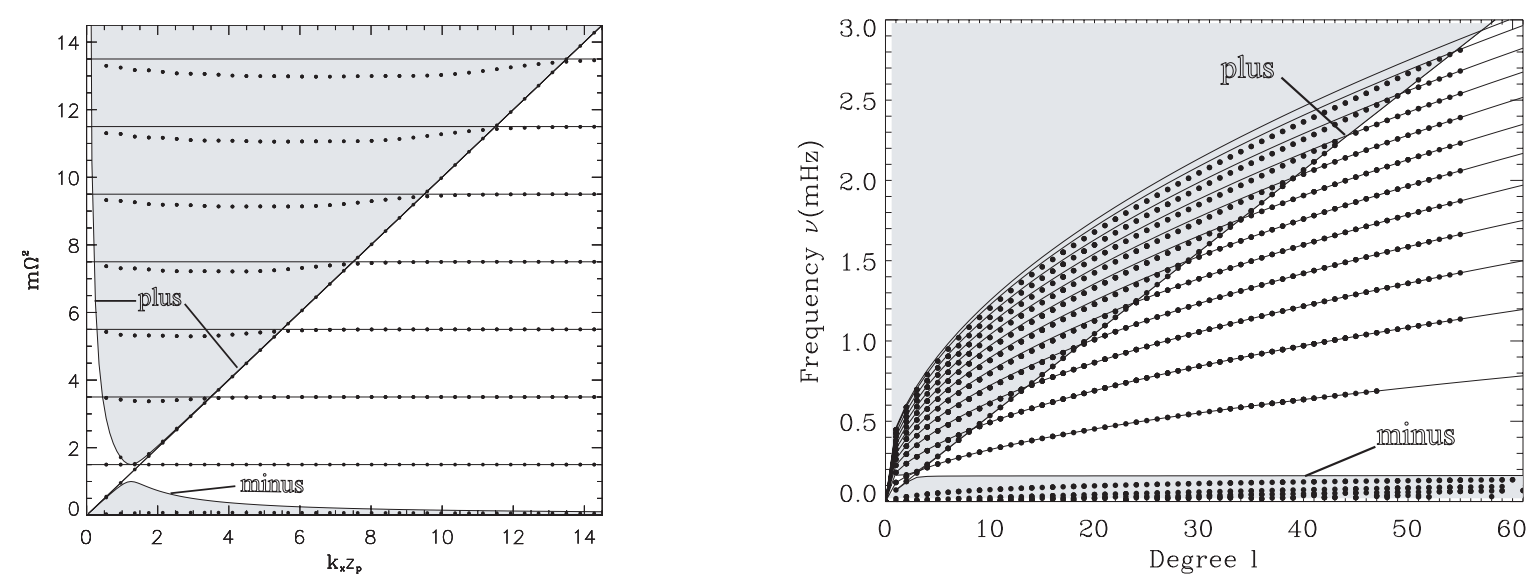

Fig. 2. Diagnostic diagrams for the tachocline model (left) normalised $\left(m \Omega^{2}\right.$ against $\left.k_{x} z_{\mathrm{p}}\right)$ and (right) dimensional (cyclic frequency $v$ against degree $l$ ). In both types of diagram, each full circle represents a mode of degree $l$, where $l$ has been increased from the degree $l=1$. The "plus" and "minus" cut-off frequencies, $\omega_{+}$and $\omega_{-}$, separate the shaded domain of propagation from the unshaded domain of evanescence in the isothermal layer. The limits of the range examined, $0.1 \leq \Omega^{2} \leq 15$, are indicated by thin lines in the dimensional diagram.

The roots are deflected from the ridges as the degree decreases and in particular close to the Lamb dispersion line. The modes for a model with magnetic field differ slightly from its field-free counterpart, but the diagnostic diagrams are indistinguishable. The tiny frequency shifts which one can obtain by comparing the two are the object of the following discussion. But, it may be noted at this point that the shifts are more prominent close to the Lamb dispersion line and that the shifts peak mostly on the oscillatory side of the Lamb separatrix.

The frequency shifts considered are generally the cyclic frequency difference $\Delta v_{n l}\left(=\Delta v_{n l}\right)$, between $v_{n l}(\max )$ obtained with a magnetic field and $v_{n l}(\mathrm{~min})$ in the absence of a magnetic field (i.e. obtained with a field-free isothermal layer); no averaging of $v$ is made. The shifts are shown in Figs. 3 and 4 for individual modes in radial order $n$ as a function of horizontal phase speed $\omega / k_{x}$, where $\omega$ is the base frequency $2 \pi v_{n l}(\mathrm{~min})$; the bottom two rows of Fig. 4 give summed results. This representation is helpful when comparing our results with a perturbation theory approach, which is explored in Appendix C. Modes with turning points located within the isothermal layer, at depths $z_{\mathrm{t}}$ between $z_{\mathrm{p}}$ and $z_{\mathrm{q}}$, have an horizontal phase speed given by the sound speed $c_{\mathrm{c}}$ in the field-free layer and indicated as a vertical dashed line in Figs. 3 and 4. For modes with turning points outside the layer, the turning depth goes as the square of the horizontal phase speed (see Fig. 1). From the summed results of Fig. 4, one can see that the amplitude of the shifts culminates for modes with horizontal phase speeds slightly above the sound speed $c_{\mathrm{c}}$.

In the long wavelength approximation $(\kappa h \ll 1)$, the effect of the modified background equilibrium is balanced by the thickness of the layer (see Eq. (49)) and in the case of parallel propagation $\left(k_{y}=0\right)$, Eq. (47) is then

$\frac{\Delta_{\perp}}{\Delta} \sim\left(1-\frac{k_{x}^{2} c_{\mathrm{c}}^{2}}{\omega^{2}}\right)\left(1-\frac{1}{1+\frac{\omega^{2}}{k_{x}^{2}}\left(\frac{1}{h g}-\frac{1}{2 H_{0} g}\right)}\right)$.

The sign of $\Delta_{\perp} / \Delta$ depends also whether $\omega^{2}>k_{x}^{2} c_{\mathrm{c}}^{2}$ or $\omega^{2}<$ $k_{x}^{2} c_{\mathrm{c}}^{2}$. The right-hand side of Eq. (50) is small if $h \sim 2 H_{\mathrm{o}}$ or for frequencies around the Lamb mode $\left(\omega^{2} \approx k_{x}^{2} c_{\mathrm{c}}^{2}\right)$ as in the case of no gravity. The effect for modes with large horizontal phase speeds $\left(\omega / k_{x} \gg c_{\mathrm{c}}\right)$ would be a change in frequency that is inversely proportional to $\beta$.

For a thin layer or a weak magnetic field, the geometry is dominant, and $\Delta_{\perp} / \Delta$ does not depend much on $\beta$ but depends on $h$ : the thinner the layer is, the larger the effect. This is why for the tachocline model, which has $h \ll H_{\mathrm{o}}$, we obtain a change in frequency inversely proportional to $\beta$ (Roberts \& Campbell 1986; Campbell 1987; Daniell 1998). On the other hand, for a relatively intense magnetic field or a relatively thick layer, the wave is more affected by the modified background equilibrium in the presence of the magnetic field than by the geometry (as long as $h \gg H_{0}$ ). This concerns in particular the shallow field model, which, despite a plasma $\beta$ almost as large as in the tachocline model, marks its difference by having $h \gg H_{0}$. Then the effect of $\Delta_{\perp} / \Delta$ is the largest for modes with $\Omega^{2} \sim 2 k_{x} H_{0}$, i.e. $\frac{\omega^{2}}{k_{x}^{2}} \sim \frac{2}{\gamma} c_{\mathrm{c}}^{2}$. These modes have horizontal phase speeds slightly above the sound speed $c_{\mathrm{c}}$. In Fig. 4, for the highest radial orders in the shallow field model, the location of such a perturbation $\left(\omega / k_{x} \sim 87.6 \mathrm{~km} \mathrm{~s}^{-1}\right)$ is identified as corresponding to the highest peak in the shifts.

Returning to Eq. (50), it is possible to reduce the expression, when $\beta$ is large and $h / z_{\mathrm{p}} \lesssim 1$ (i.e. $g h \lesssim c_{\mathrm{c}}^{2}$ ) for $\omega / k_{x} \gg c_{\mathrm{c}}$, viz.

$$
\frac{\Delta_{\perp}}{\Delta} \sim 1-m \frac{h}{z_{\mathrm{p}}} \frac{k_{x}^{2} c_{\mathrm{c}}^{2}}{\omega^{2}}
$$

One can see that the effect depends now on the thickness of the magnetic layer as seen from the surface. This explains why the amplitude of the shifts decreases with an increase in the mode turning depth below the layer (or the corresponding horizontal phase speed).

Considering the individual ridges of very low degree $l$, increasing in radial order $n$ as the frequency increases, the above analytical descriptions in terms of frequency rather than horizontal phase speed is also observed in the numerical results. In Fig. 5, fractional frequency shifts $\Delta v / v$ are plotted in 

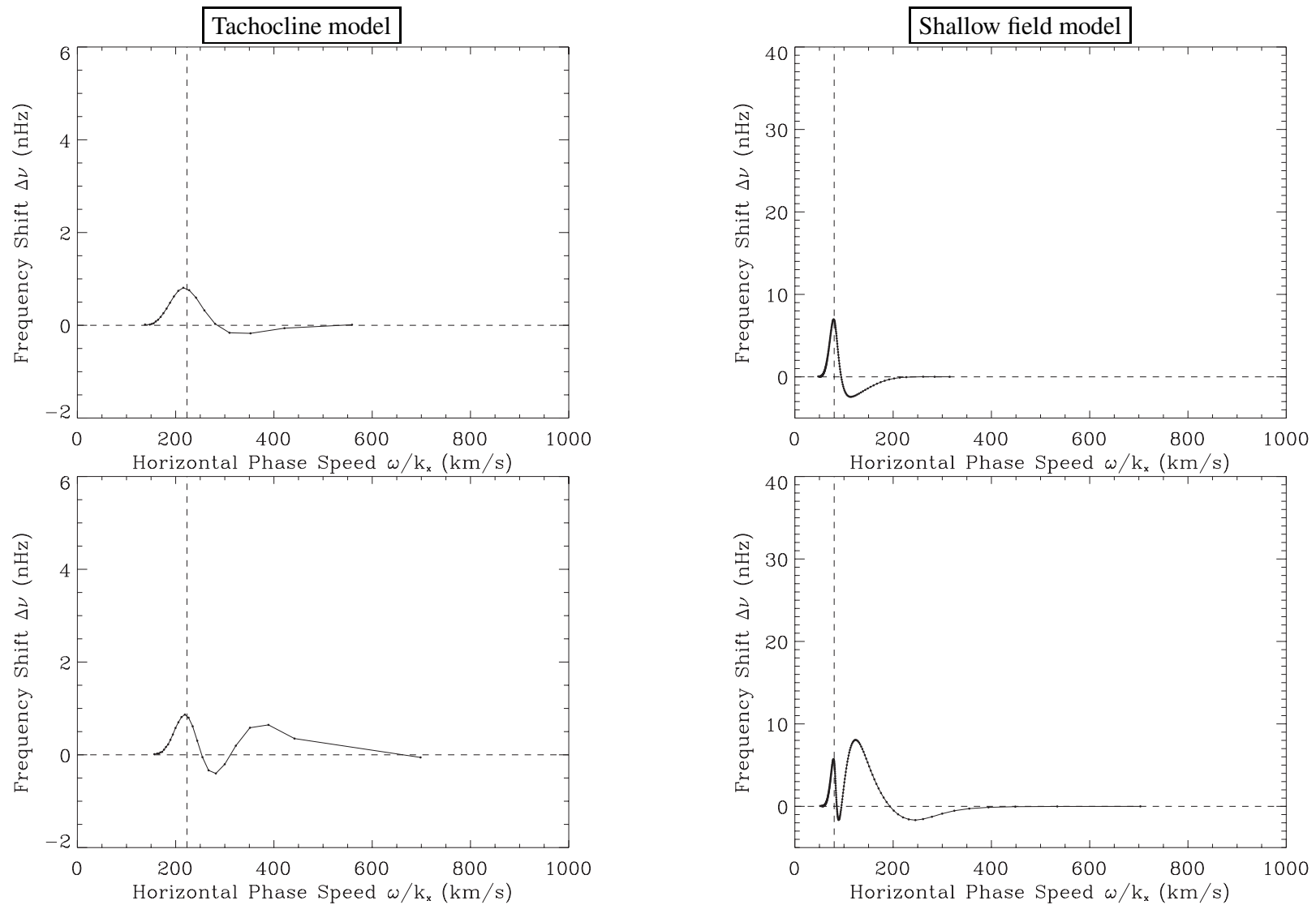

$p_{3}$
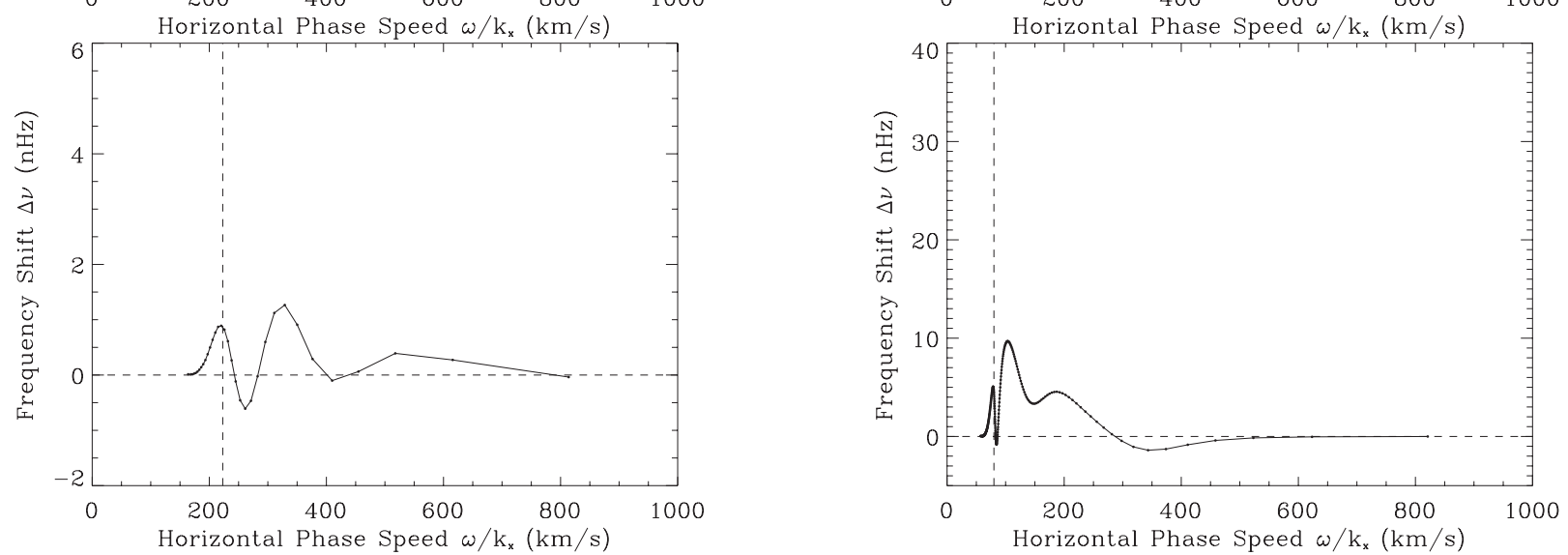

$p_{4}$
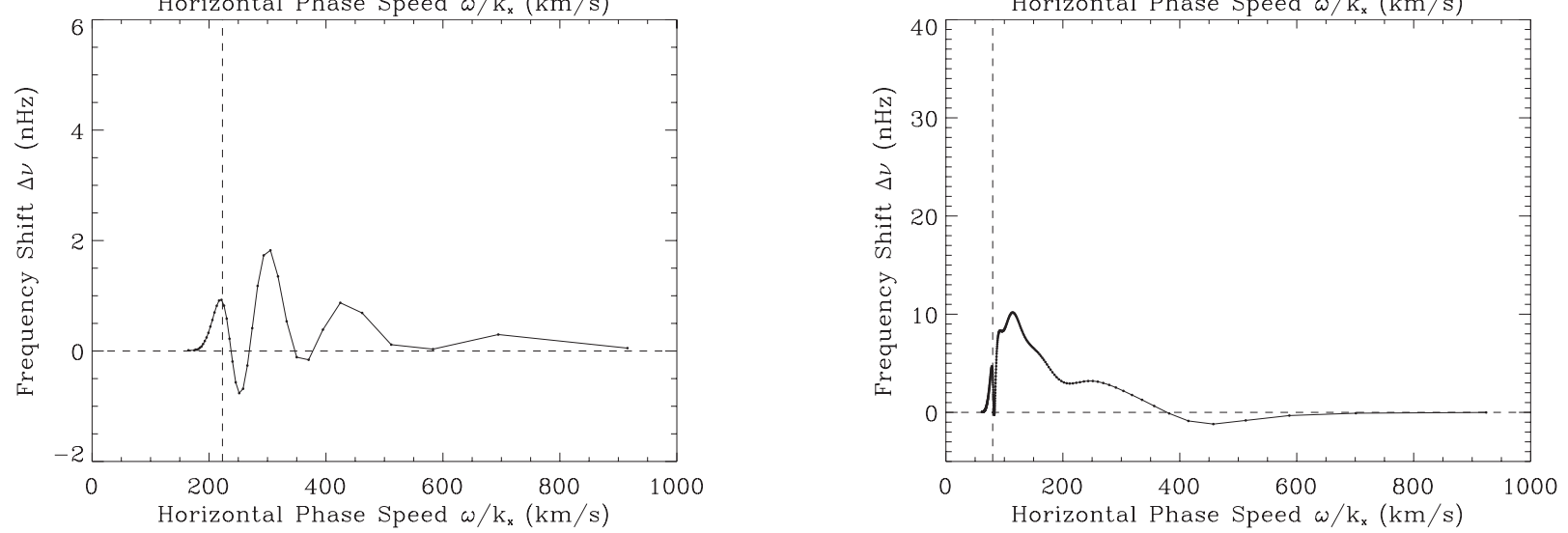

Fig. 3. The difference $\Delta v$ in cyclic frequency between frequencies $v_{n l}(\max )$ obtained with a magnetic slab and frequencies $v_{n l}(\min )$ obtained in the absence of a magnetic field. $\Delta v$ is plotted as a function of horizontal phase speed $\frac{\omega}{k_{x}}$, where $\omega$ is the base frequency $2 \pi v_{n l}(\min )$ and $k_{x}=\sqrt{l(l+1)} / R_{\odot}$, for the tachocline model (left) and the shallow field model (right) and $p$-modes of radial orders $n=1$ to $n=4$. The sound speed $c_{\mathrm{c}}$ in the magnetic layer is indicated as a vertical dashed line. 

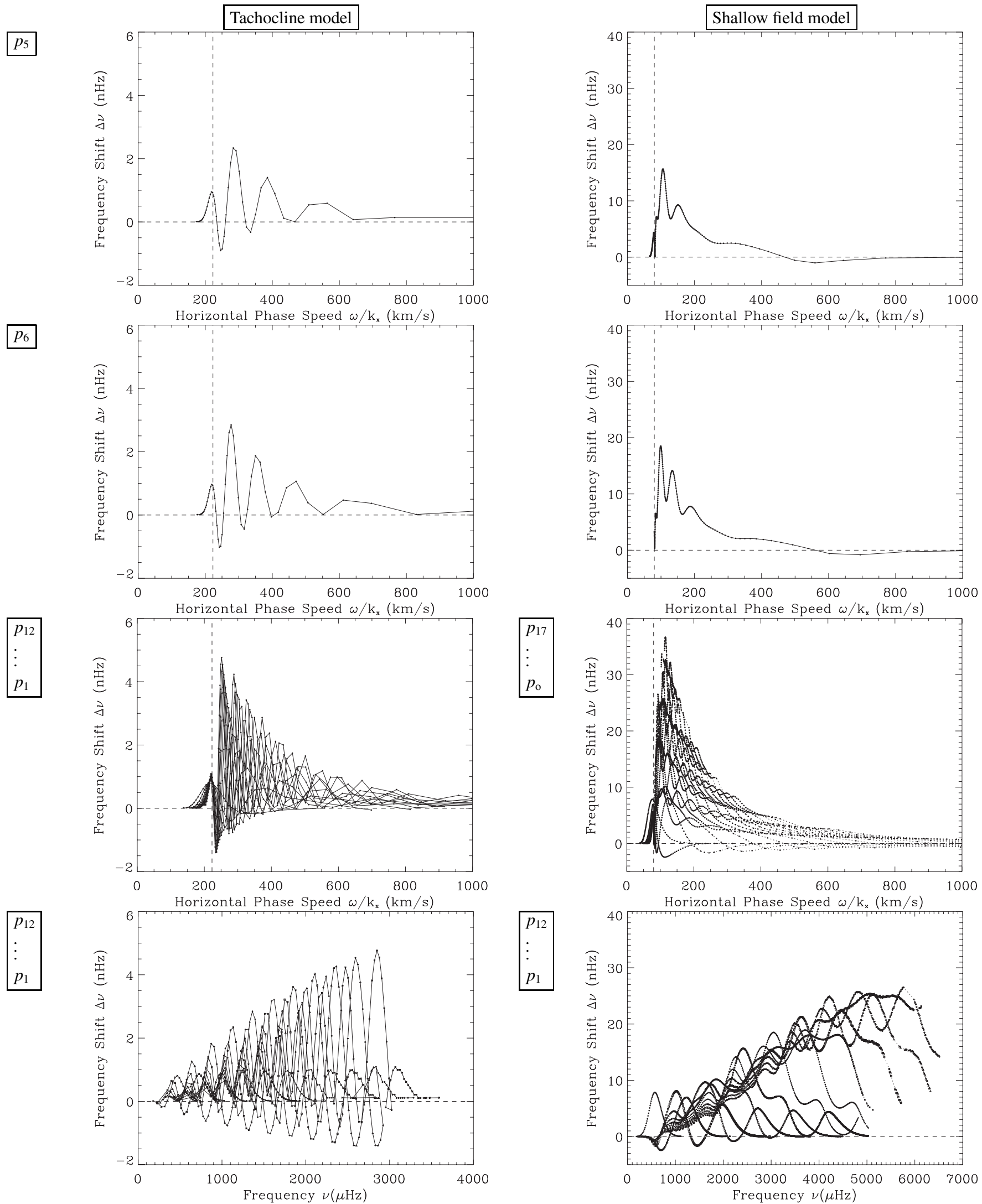

Fig. 4. As in Fig. 3, the difference $\Delta v$ as a function of horizontal phase speed $\frac{\omega}{k_{x}}$ for $p$-modes of radial orders $n=5$ and $n=6$, and for all $p$-modes with $n=1,2, \ldots, 12$ for the tachocline model or from $n=0$ to $n=17$ for the shallow field model; also shown is $\Delta v$ as a function of base frequency $v=v_{n l}(\min )$ for all $p$-modes of radial orders $n=1$ to $n=12$. 

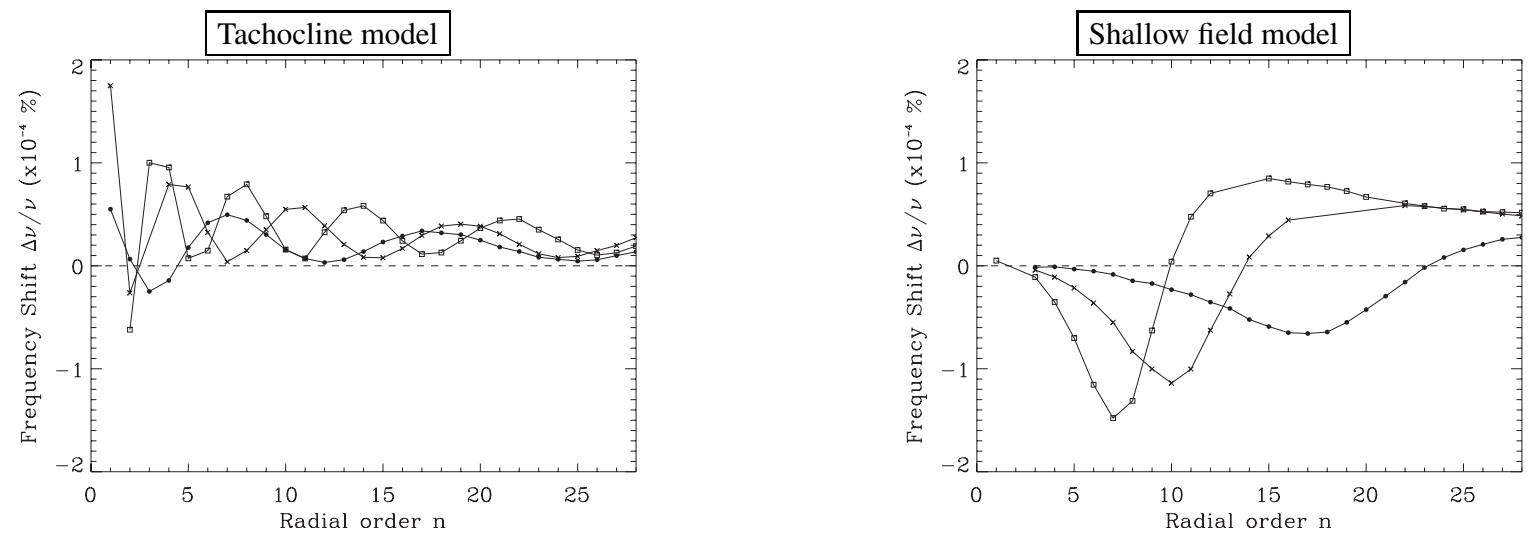

Fig. 5. Fractional percentage frequency shifts versus radial order $n$, with lines joining shifts from modes of same degree $l$, for $l=1$ (filled circles), $l=2$ (crosses) and $l=3$ (open squares); (left) is for the tachocline model and (right) is for the shallow field model.

percentages with respect to the base frequency $v(\min )$, against the radial order $n$ of the mode. At low radial order $n$, the negative dips are consistent with Eq. (51) and the difference of amplitude between the two models is explained by the difference in the ratio $z_{\mathrm{p}} / h$. As the radial order increases, the shifts become positive, again consistent with the approximate result (51).

The oscillatory patterns and their negative extrema may be interpreted as manifestations of the resulting adjustment of the nodes at the interfaces of the layer. It is interesting to note that these results are consistent with numerical results by Gough \& Thompson (1990), who noticed an "irregular" behaviour of central frequency shifts for modes with degree $l=2$ and stated that "with better sampling of points these results would exhibit an oscillatory behaviour" (see their Fig. 14b and last paragraph in Sect. 7 of Gough \& Thompson 1990). Recent observations perhaps indicate the existence of such an oscillatory pattern in low- $l$ shifts (Jiménez-Reyes et al. 2001). These new details need further investigation, but if confirmed they may prove of interest for linking theoretical interpretations to the observations.

For modes with turning points above the layer, the first peaks in the shifts of successive $p$-modes mark the separation between evanescent and oscillatory behaviour of the $p$-mode inside the layer. This shows that a given mode which propagates in the solar interior, with a cavity depth above the perturbative layer, may still be affected by the presence of the layer, through its evanescent tail sweeping the lower region. The acoustic oscillations are modulated by changing conditions in a region in which the waves are evanescent.

\section{Analytical approximation}

The analytical approximation to the dispersion relation derived below proves useful as an insight into the numerical results. Also, the approximated shifts turn out to have properties that reproduce the main shape of the oscillatory pattern in the shifts obtained numerically. This property of the approximation offers encouragement to deduce analytically shifts for modes with perpendicular propagation.

\subsection{The hydrodynamical effect of a thin isothermal layer}

Consider first the hydrodynamical effect of a thin nonmagnetised isothermal layer. The dispersion relation (36) is simplified for a field-free $(\beta \rightarrow \infty)$ isothermal slab to

$\epsilon_{\mathrm{p}}^{-} \epsilon_{\mathrm{q}}^{+} \mathrm{e}^{2 \kappa h}-\epsilon_{\mathrm{p}}^{+} \epsilon_{\mathrm{q}}^{-}=0$,

where

$\epsilon_{\mathrm{p}}^{ \pm}=\left(k_{\mathrm{h}}+\Lambda^{ \pm}\right) M-2 k_{\mathrm{h}} M^{\prime}, \quad \epsilon_{\mathrm{q}}^{ \pm}=\left(k_{\mathrm{h}}+\Lambda^{ \pm}\right) U-2 k_{\mathrm{h}} U^{\prime}$

$\kappa$ is real for surface modes and imaginary for oscillatory modes in the layer. Two first order approximations are applied.

First, the isothermal layer of thickness $h=z_{\mathrm{q}}-z_{\mathrm{p}}$ is considered to be thin so that $h \ll z_{\mathrm{p}}$. Then $\mathrm{e}^{2 \kappa h} \approx 1+2 \kappa h$, and the dispersion relation can be expanded in the form

$D \approx D_{0}+\frac{h}{z_{\mathrm{p}}} D_{1}$

where $D_{0}$ corresponds to the dispersion relation for a model without an isothermal layer (i.e., a simple polytrope with $p$-mode solutions given by Eq. (13)) and $D_{1}$ is the first order correction to that dispersion relation in the presence of an isothermal layer of thickness $h$.

The eigenfrequency $\omega$ is the result of the effect of this thin layer on the original eigenfrequency $\omega_{0}$, so that $\omega=\omega_{0}+\Delta \omega$. The second analytical approximation comes from assuming a priori that the frequency change is small, so that $\Delta \omega \ll \omega$, a result found numerically. Then, a first order Taylor expansion can be applied to the $D_{0}$ term around the base frequency $\omega_{0}$, viz.

$D_{0}(\omega) \approx D_{0}\left(\omega_{0}\right)+D_{0}^{\prime}\left(\omega_{0}\right) \Delta \omega$.

Since $D(\omega)=0$ and $D_{0}\left(\omega_{0}\right)=0$, Eqs. (54) and (55) combine to give an analytical approximation to the frequency shift, viz.

$\Delta \omega \approx-\frac{h}{z_{\mathrm{p}}} \frac{D_{1}(\omega)}{D_{0}^{\prime}\left(\omega_{0}\right)} \approx-\frac{h}{z_{\mathrm{p}}} \frac{D_{1}\left(\omega_{0}\right)}{D_{0}^{\prime}\left(\omega_{0}\right)}$.

Thus, $\Delta \omega$ is directly proportional to the thickness $h$ of the layer. 

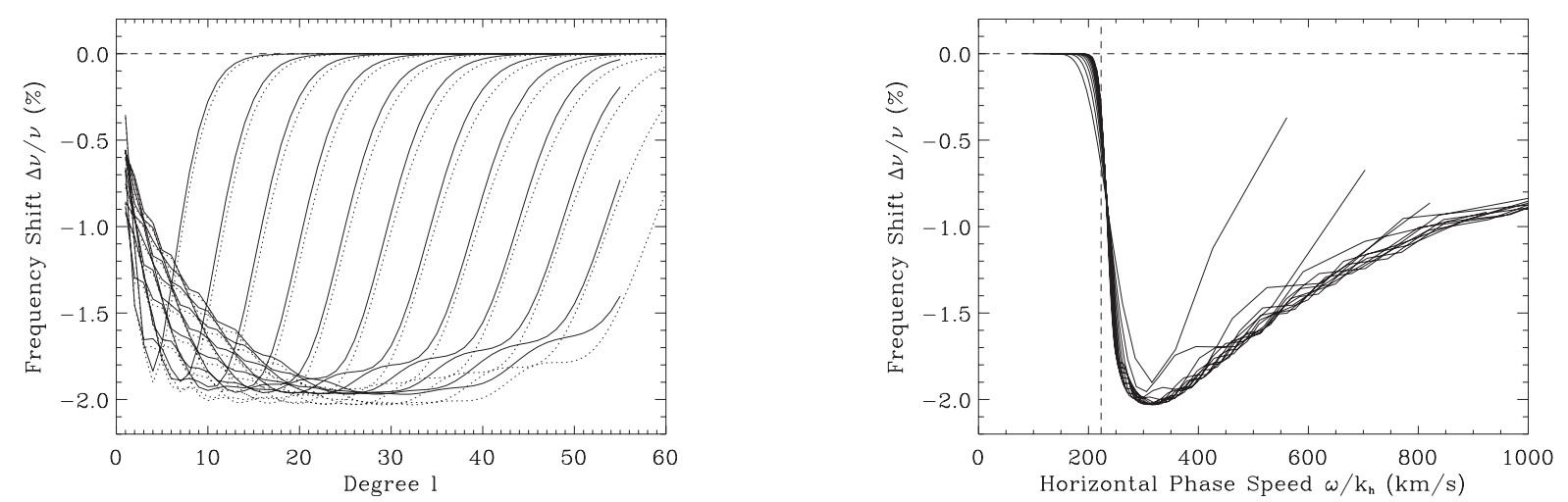

Fig. 6. (Left) Percentages of fractional frequency shifts versus degree $l$, due to the hydrodynamical effect of a buried layer below the convection zone. Numerical and analytical results are shown respectively in bold and dotted lines, joining shifts of the same radial order $n$, from $n=1$ (confined to low $l$ ) to $n=12$ (seen at higher $l$ ). (Right) The analytical results are determined frequency shifts as a function of horizontal phase speed $\omega / k_{\mathrm{h}}$.

\section{From}

$D(\omega)=\frac{\epsilon_{\mathrm{p}}^{-} \epsilon_{\mathrm{q}}^{+} \mathrm{e}^{2 \kappa h}-\epsilon_{\mathrm{p}}^{+} \epsilon_{\mathrm{q}}^{-}}{4 k_{\mathrm{h}} \kappa}=0$,

the terms $D_{0}$ and $D_{1}$ are

$D_{0}=\frac{\epsilon_{\mathrm{p}}^{-} \epsilon_{\mathrm{q}}^{+}-\epsilon_{\mathrm{p}}^{+} \epsilon_{\mathrm{q}}^{-}}{4 k_{\mathrm{h}} K}, \quad D_{1}=z_{\mathrm{p}} \frac{\epsilon_{\mathrm{p}}^{-} \epsilon_{\mathrm{q}}^{+}}{2 k_{\mathrm{h}}}$.

Developing $D_{0}$ yields

$D_{0}=M U^{\prime}-U M^{\prime}=-\frac{\Gamma(m+2)}{\Gamma(-\Upsilon)} \frac{\mathrm{e}^{2 k_{\mathrm{h}} z_{\mathrm{p}}}}{\left(2 k_{\mathrm{h}} z_{\mathrm{p}}\right)^{m+2}}$,

where $\Upsilon=\left[m \Omega^{2}-(m+2)\right] / 2$. When taking the first derivative of $D_{0}$ with respect to $\omega$, only the term in $\Upsilon$ is variable. Writing $u=-\Upsilon$, it is necessary to consider

$\frac{\partial \frac{1}{\Gamma(u)}}{\partial u}=-\frac{\Gamma^{\prime}(u)}{(\Gamma(u))^{2}}=-\frac{\Psi(u)}{\Gamma(u)}$.

This derivative must be estimated around $u \approx 1-n$, where $n$ is a non-zero and positive integer. For such points, the functions $\Psi$ and $\Gamma$ are two poles. The problem is solved by applying reflection formulae, so that (see Foullon 2002a, for details)

$\lim _{u \rightarrow 1-n} \frac{\partial \frac{1}{\Gamma(u)}}{\partial u}=(-1)^{n-1}(n-1) !$.

This finally gives the derivative of $D_{0}$ with respect to $\omega$ evaluated at $\omega_{0}$,

$D_{0}^{\prime}\left(\omega_{0}\right)=\frac{m \omega_{0}}{g k_{\mathrm{h}}}(-1)^{n-1}(n-1) ! \Gamma(m+2) \frac{\mathrm{e}^{2 k_{\mathrm{h}} z_{\mathrm{p}}}}{\left(2 k_{\mathrm{h}} z_{\mathrm{p}}\right)^{m+2}}$.

In $D_{1}\left(\omega_{0}\right)$, the confluent hypergeometric functions can be simplified in terms of generalised Laguerre polynomials.

After some algebra, the fractional frequency shift is determined as

$\frac{\Delta v}{v}=-\frac{h}{k_{\mathrm{h}}} \cdot \frac{\left(2 k_{\mathrm{h}} z_{\mathrm{p}}\right)^{m+2}}{\mathrm{e}^{2 k_{\mathrm{h}} z_{\mathrm{p}}}} \cdot \frac{\operatorname{Re}(\Pi)\left(L_{1}\right)^{2}(n-1) !}{2 m\left(1+\frac{2 n}{m}\right) \Gamma(n+m+1)}$,

where

$\Pi=\left[\left(1+2 \frac{L_{2}}{L_{1}}\right) k_{\mathrm{h}}-\frac{1}{2 H_{\mathrm{o}}}-\kappa\right] \cdot\left[\left(1+2 \frac{L_{2}}{L_{1}}\right) k_{\mathrm{h}}-\frac{1}{2 H_{\mathrm{o}}}+\kappa\right](64)$ and $L_{1}, L_{2}$ are generalised Laguerre polynomials

$$
L_{1}=L_{n-1}^{m+1}\left(2 k_{\mathrm{h}} z_{\mathrm{p}}\right), \quad L_{2}=L_{n-2}^{m+2}\left(2 k_{\mathrm{h}} z_{\mathrm{p}}\right) .
$$

These polynomials and Eq. (63) were evaluated with the mathematical software Mathematica. As shown in Fig. 6, the analytical approximation to account for the presence of a thin isothermal field-free layer in a polytrope $\left(h=0.05 R_{\odot}\right.$ and $z_{\mathrm{p}} \approx 0.39 R_{\odot}$ ) confirms the shape and the magnitude of the shifts obtained numerically.

\subsection{The magnetohydrodynamical effect of a thin isothermal layer}

The numerical results contain tiny shifts, not discernible on the graph, due only to the presence of a magnetic field $(\beta \approx 12236$ for $\left.B_{\mathrm{o}}\left(z_{\mathrm{p}}\right)=0.3 \mathrm{MG}\right)$. These effects are difficult to determine analytically from Eq. (36). However, by considering the case of a field-free layer we may provide a useful analytical estimate of the frequency shifts. To do this we replace the layer terms $H_{\mathrm{o}}$ and $\kappa$ in Eq. (64) by their magnetic counterparts given in Table 2. The result is an approximation to the shifts between the model including the magnetic layer and the simple polytrope. Subtracting from them the analytical shifts due to the field-free layer, one can find the magnitude of these tiny shifts, as shown in Fig. 7 for parallel propagation (Foullon 2001). Because our approximation is based on the dispersion relation for a field-free slab (Eq. (52)) and not on the dispersion relation (Eq. (36)) yielding the numerical results, we do not expect the shifts derived analytically by this process to match exactly the form found from solving the full dispersion relation numerically. However, we do expect the magnitude of the shifts to be similar. In Fig. 7, the analytical results confirm the magnitude of the numerical results for modes propagating immediately below the layer, which yield the largest shifts, and for modes propagating beyond. There is not such a good agreement for modes yielding lower shifts, propagating above and inside the magnetic layer.

For realistic parameters in the solar interior, the sole effect of a magnetic field on central $p$-mode frequencies is negligible for parallel propagation: $\Delta v / v \sim 2 \times 10^{-6}$ for the tachocline 

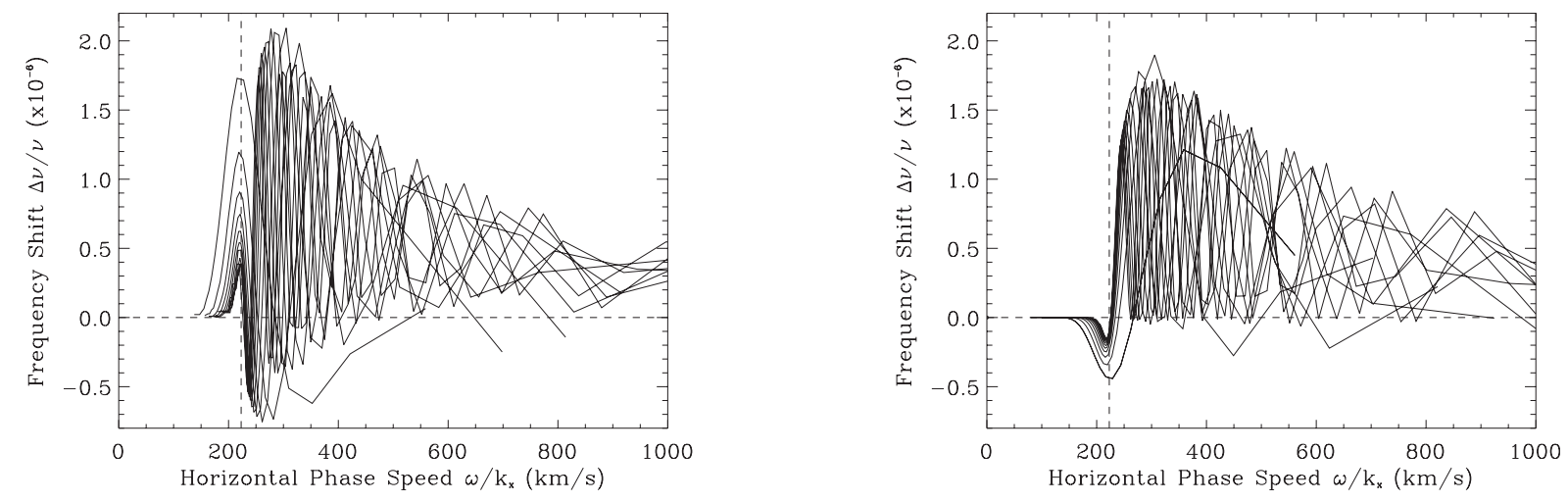

Fig. 7. Fractional frequency shifts versus horizontal phase speed $\omega / k_{x}$, due to the sole effect of a magnetic field in a buried layer below the convection zone $\left(B_{\mathrm{o}}\left(z_{\mathrm{p}}\right)=0.3 \mathrm{MG}\right)$, for parallel propagation $\left(k_{y}=0\right)$ : (left $)$ numerical and $($ right $)$ analytical results are shown by lines joining shifts of the same radial order $n$, from $n=1$ to $n=11$.
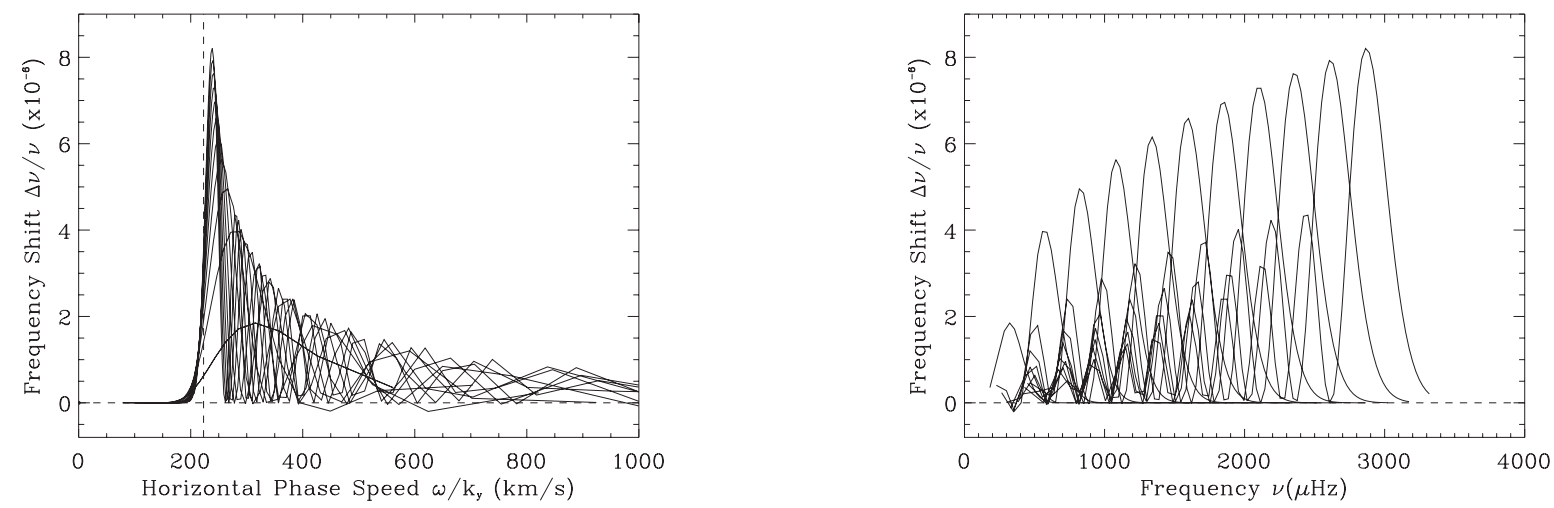

Fig. 8. Fractional frequency shifts due to the sole effect of a magnetic field in a buried layer below the convection zone $\left(B_{0}\left(z_{\mathrm{p}}\right)=0.3 \mathrm{MG}\right)$, for perpendicular propagation $\left(k_{x}=0\right)$. The shifts are shown by lines joining shifts of the same radial order, from $n=1$ to $n=11$, and plotted as a function of (left) the horizontal phase speed $\omega / k_{y}$ or (right) the base frequency.

model, which includes a buried layer of thickness $h=0.05 R_{\odot}$, with maximum field strength $B_{\mathrm{o}}\left(z_{\mathrm{p}}\right)=0.3 \mathrm{MG} ; \Delta v / v \sim 10^{-5}$ for a shallow layer of same thickness at $z_{\mathrm{p}} \approx 0.05 R_{\odot}$ for $B_{\mathrm{o}}\left(z_{\mathrm{p}}\right)=30 \mathrm{kG}$ (with $\beta \approx 12176$ ). These shifts are to be compared with the observations, $\Delta v / v \sim 2 \times 10^{-4}$ over a solar cycle.

Moreover, for the tachocline model, the relative effect on $p$-mode frequencies of a buried magnetic layer is much larger for waves propagating perpendicular rather than parallel to the magnetic field lines (see Fig. 8).

As the solar cycle rises, the $x$ and $y$-directions are presumed to flip by 90 degrees - the field lines passing from poloidal to toroidal at the base of the convection zone (see, e.g., Dikpati \& Charbonneau 1999). Thus, instead of searching for an explanation through a variation in field strength, one may also interpret the observed shifts in global frequencies by a change in orientation of the magnetic field lines (Foullon 2002b). At some reference time in the solar cycle, the "global" waves (i.e. as if the Sun were not rotating) propagate perpendicular to a toroidal field; at a time in opposite phase, they propagate parallel to a poloidal one. This change of configuration would produce maximum relative frequency shifts almost as large as the sole effect of the field strength on perpendicularly propagating waves (see Fig. 9). These last results lead essentially to the conclusion that at the base of the convection zone the presumed cyclical change of orientation of magnetic field lines has a larger effect on global mode frequencies than an increase in field strength alone would have on modes propagating parallel to the magnetic field lines. However, an effect as large as $\Delta v / v \sim 9 \times 10^{-6}$ at the peak shift frequency $3900 \mu \mathrm{Hz}$ (see right diagram of Fig. 8) is still negligible in comparison to the observations.

The analytical approximation to the frequency shifts cannot be applied to the shallow field model, for which $h \approx z_{\mathrm{p}}$. It is likely that, as for the tachocline model, shifts for perpendicular propagation would be larger than for parallel propagation. One should then rely on numerical methods to assess whether the sunspot's anchoring zone could contribute efficiently to the global frequency shifts observed over the solar activity cycle. However, more careful modelling is needed: it would be relevant to include the superadiabatic gradient below the surface as well as an atmosphere above the surface; these effects are likely to be more crucial for the shallow field model.

\section{Discussion and conclusions}

\subsection{On the choice of the constant value of gravitational acceleration}

The depth of the base of the convection zone $z_{\mathrm{p}}$ has a nonnegligible effect on the frequency shifts, but does not have a straight-forward proportional dependence, as illustrated by the analytical approximation (63). However, for parallel propagation, $z_{\mathrm{p}}$ appears in the dispersion relation through the 


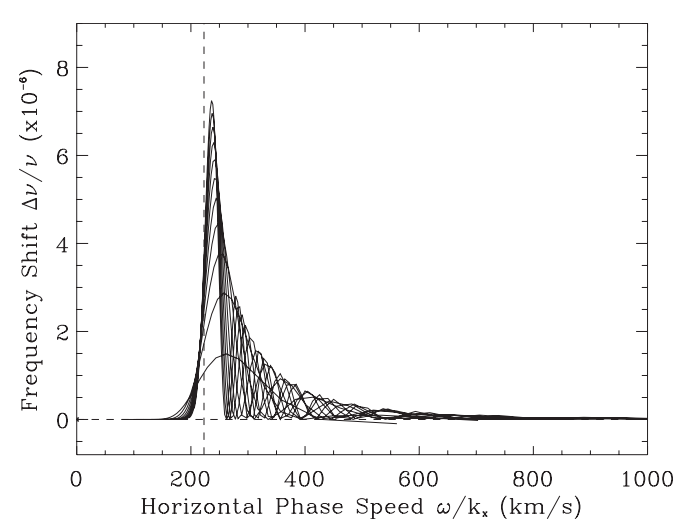

Fig. 9. Fractional frequency shifts versus horizontal phase speed, due to the effect of perpendicular propagation $\left(k_{x}=0\right)$ with respect to parallel propagation $\left(k_{y}=0\right)$, for a magnetic field at the base of the convection zone $\left(B_{\mathrm{o}}\left(z_{\mathrm{p}}\right)=0.3 \mathrm{MG}\right)$, are shown by lines joining shifts of the same radial order $n$, from $n=1$ to $n=11$.

wavenumber combination $k_{x} z_{\mathrm{p}}$ and in the ratio $h / z_{\mathrm{p}}$. The roots obtained numerically are the normalised eigenfrequencies $\Omega^{2}=\omega^{2} /\left(g k_{x}\right)$, and $g$ is not directly specified in the dispersion relation. Without changing the value of the sound speed at the base of the convection zone, viz. $c_{\mathrm{c}}=\frac{g z_{\mathrm{p}}}{m}=223 \mathrm{~km} \mathrm{~s}^{-1}$, nor the ratio $h / z_{\mathrm{p}} \sim 0.125$, it is therefore possible to obtain results for a new tachocline model just by scaling the numerical results through a change in $g$, where $z_{\mathrm{p}}$ and $h$ are scaled accordingly. If $\alpha$ is the scaling factor between two different values for the gravitational acceleration, denoted by the subscripts 1 and 2, viz. $(g)_{2}=\alpha(g)_{1}$, then the conditions that

$$
\begin{aligned}
& \left(g z_{\mathrm{p}}\right)_{2}=\left(g z_{\mathrm{p}}\right)_{1}=\left[\alpha(g)_{1}\right]\left[\frac{\left(z_{\mathrm{p}}\right)_{1}}{\alpha}\right], \\
& \left(k_{x} z_{\mathrm{p}}\right)_{2}=\left(k_{x} z_{\mathrm{p}}\right)_{1}=\left[\alpha\left(k_{x}\right)_{1}\right]\left[\frac{\left(z_{\mathrm{p}}\right)_{1}}{\alpha}\right]
\end{aligned}
$$

lead to $(\Delta \omega)_{2}=\alpha(\Delta \omega)_{1}$.

If $g=530 \mathrm{~m} \mathrm{~s}^{-2}$ (Basu et al. 1994, p. 214), the scale factor is almost two $(\alpha \sim 1.934) ; z_{\mathrm{p}} \sim 0.202 R_{\odot}$ and $h \sim 0.026 R_{\odot}$. The resulting shifts are shown in Fig. 10. Comparing with Fig. 4, one can see that although the layer is almost half as wide the change in $g$ from $274 \mathrm{~m} \mathrm{~s}^{-2}$ to $530 \mathrm{~m} \mathrm{~s}^{-2}$ produces shifts that are almost twice as large, and the change in $z_{\mathrm{p}}$ from $0.391 R_{\odot}$ to $0.202 R_{\odot}$ extends the shifts from a range of degree $l=0$ to $l=70$, doubled to $l=140$. This points to the need to consider a spherical geometry treatment with non-uniform gravitational acceleration.

\subsection{Solar buried magnetic layer}

We have examined the influence of a buried magnetic layer. The horizontal layer of field is located either at the base of the convection zone or higher up at the supposed anchoring zone for sunspots. In either case, frequency shifts due to an evolving buried magnetic field are found to be of order $\Delta v / v=$ $(2-10) \times 10^{-6}$, much smaller than the observed shifts over the solar cycle $\left(\Delta v / v=2 \times 10^{-4}\right)$. Shifts due to changes in orientation of the field are found to be larger than those brought about by field strength changes. Nonetheless, buried magnetic

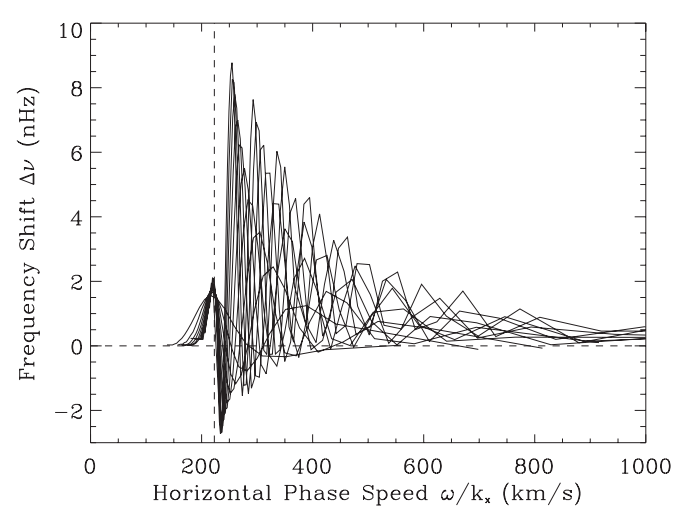

Fig. 10. Frequency shifts for $p$-modes propagating parallel to the magnetic field lines of the tachocline model (with $B_{\mathrm{o}}\left(z_{\mathrm{p}}\right)=0.3 \mathrm{MG}$ ), but with $g=530 \mathrm{~m} \mathrm{~s}^{-2}, h \sim 0.026 R_{\odot}$ and $z_{\mathrm{p}} \sim 0.202 R_{\odot}$.

fields are unable to produce frequency shifts that are comparable with those observed. Consequently, it would appear that other effects, such as the influence of the magnetic atmosphere (e.g., Campbell \& Roberts 1989; Evans \& Roberts 1992; Jain \& Roberts 1996), are more significant.

There are grounds for inferring the signature of the buried layers through examining shifts of various degree. Several aspects may be distinguished. First, the $p$-mode frequencies are increased proportionally to $1 / \beta$, where $\beta$ is the squared ratio between the sound and Alfvén speeds in the magnetic layer. This effect may be directly related to the Lagrangian rate of change in magnetic pressure perturbation, which compresses the volume perpendicular to the magnetic field lines. When the plasma $\beta$ is large, one can say approximately that magnetism acts only through the dynamics (the magnetic pressure perturbation), but in general the effect is more subtle because the magnetic field affects also the equilibrium pressure distribution. This second aspect involves the variation of the vertical velocity amplitude, which approximately increases on the spatial scale of the layer thickness and decreases on a scale of two (magnetically-modified) density or pressure scale-heights. In particular, when the plasma $\beta$ is large, it may be said that the frequency changes depend upon the layer thickness "as seen from the surface".

The form of the dispersion relation derived proved useful in obtaining an analytical approximation to the effect of the buried layer in a polytrope. This analytical approximation was consistent with the numerical results for parallel propagation and served to evaluate the amplitude of the shifts for perpendicular propagation. For realistic solar parameters at the base of the convection zone (with maximum field strength $B_{\mathrm{o}}=0.3 \mathrm{MG}$ ), the relative effect on $p$-mode frequencies of a buried magnetic layer of thickness $h=0.05 R_{\odot}$ is much larger for waves propagating perpendicular rather than parallel to the magnetic field lines. These last results opened a new possibility to obtain frequency shifts, not through a variation in magnetic field strength as usually explained, but by a change in orientation of the magnetic field lines. For a change of magnetic topology at the base of the convection zone, it is expected that the "global" waves (i.e. as if the Sun were not rotating) would propagate perpendicular to a toroidal field at a time which was out of phase with the maximum of solar activity seen at the surface, and 
that they would propagate parallel to a poloidal field at a time with about the same phase difference with solar minimum. This change of configuration would produce frequency shifts anticorrelated or out of phase with the solar cycle. Nonetheless, for the tachocline model, an effect as large as $\Delta v / v \sim 9 \times 10^{-6}$ at the peak shift frequency $3900 \mu \mathrm{Hz}$ is still negligible in comparison to the observed fractional frequency shift $\left(\Delta v / v \sim 2 \times 10^{-4}\right.$ over a solar cycle), suggesting that the observed shifts are much more likely to be explained by the influence of the changing chromospheric and coronal atmosphere. However, the sunspot anchoring zone might be relevant, and a more thorough modelling of this region is needed.

\subsection{Advantages and potentials}

1. Our numerical and analytical results show that the amplitude of the shifts culminates for modes with horizontal phase speeds slightly above the sound speed in the field-free layer. For modes with increasing turning depths below the layer, the amplitude of the shifts is expected to decrease. These properties of the frequency shifts are consistent with a perturbation theory approach, as shown in Appendix C. However, in contrast, our results show the details of an oscillatory pattern in the shifts, which may prove of interest to explain observations of similar patterns in low- $l$ shifts (Jiménez-Reyes et al. 2001). Moreover, we have shown that, like the chromosphere and even the corona, the buried layers are able to influence the properties of $p$-modes with turning points above the magnetic layer, by acting on the evanescent tail of the modes.

2 . Through analytical derivations, we have obtained agreement between our numerical and analytical results, reproducing quantitative estimates of the frequency shifts. Unlike the perturbation theory approach, our method is applicable to low degree modes. Our analytical approach provides a useful alternative to this theory.

3. Modelling similar to that presented here could also be developed for astrophysical plasmas. In particular, $p$ - and $g$-modes in polytropic accretion disks (Korycansky \& Pringle 1995), and the effect of an isothermal atmosphere that joins smoothly on to the underlying polytropic layer, may be explored (Ogilvie \& Lubow 1999). Besides, the analytical approach developed in the linear theory provides a basis for the study of nonlinear effects. It could then be extended to other stars where magnetic effects are significant (e.g. Ap stars).

Acknowledgements. C.F. acknowledges the financial support provided through the University of St Andrews, the UK Particle Physics and Astronomy Research Council and the European Community's Human Potential Programme under contract HPRN-CT-2000-00153, PLATON. We are grateful to the referee for constructive comments.

\section{References}

Antia, H. M., \& Basu, S. 2000, ApJ, 541, 442

Antia, H. M., Chitre, S. M., \& Thompson, M. J. 2000, A\&A, 360, 335

Basu, S. 1997, MNRAS, 288, 572

Basu, S., Antia, H. M., \& Narasimba, D. 1994, MNRAS, 267, 209

Bogdan, T. J., \& Zweibel, E. G. 1985, ApJ, 298, 867

Campbell, W. R. 1987, Ph.D. Thesis, University of St Andrews, Scotland
Campbell, W. R., \& Roberts, B. 1989, ApJ, 338, 538

Chaplin, W. J., Elsworth, Y., Isaak, G. R., et al. 1998, MNRAS, 300, 1077

Choudhuri, A. R., \& Gilman, P. A. 1987, ApJ, 316, 788

Christensen-Dalsgaard, J. 1980, MNRAS, 190, 765

Christensen-Dalsgaard, J., Gough, D. O., \& Thompson, M. J. 1991, ApJ, 378, 413

Daniell, M. 1998, Ph.D. Thesis, University of St Andrews, Scotland

Deubner, F., \& Gough, D. 1984, ARA\&A, 22, 593

Dikpati, M., \& Charbonneau, P. 1999, ApJ, 518, 508

Dziembowski, W. A., Goode, P. R., Kosovichev, A. G., \& Schou, J. 2000, ApJ, 537, 1026

Evans, D. J., \& Roberts, B. 1990, ApJ, 356, 704

Evans, D. J., \& Roberts, B. 1992, Nature, 355, 230

Fisher, G. H., Fan, Y., Longcope, D. W., Linton, M. G., \& Abbett, W. P. 2000, Phys. Plasmas, 7, 2173

Foullon, C. 2001, in INTAS Workshop on MHD Waves in Astrophysical Plasmas, ed. J. L. Ballester \& B. Roberts, 115

Foullon, C. 2002a, Ph.D. Thesis, University of St Andrews, Scotland

Foullon, C. 2002b, in Radial and Nonradial Pulsations as Probes of Stellar Physics, ed. C. Aerts, T. Bedding \& J. ChristensenDalsgaard, IAU Coll. 185, 482

Goedbloed, J. P. 1971, Physica, 53, 412

Goldreich, P., Murray, N., Willette, G., \& Kumar, P. 1991, ApJ, 370, 752

Gough, D. O., \& Thompson, M. J. 1990, MNRAS, 242, 25

Guenther, D. B., Demarque, P., Kim, Y.-C., \& Pinsonneault, M. H. 1992, ApJ, 387, 372

Hathaway, D., Gilman, P., Harvey, J. W., et al. 1996, Science, 272, 1306

Howe, R., Christensen-Dalsgaard, J., Hill, F., et al. 2000, ApJ, 533, L163

Howe, R., Komm, R., \& Hill, F. 1999, ApJ, 524, 1084

Jain, R., \& Roberts, B. 1994, Sol. Phys., 152, 261

Jain, R., \& Roberts, B. 1996, ApJ, 456, 399

Jiménez-Reyes, S. J., Corbard, T., Pallé, P. L., Roca Cortés, T., \& Tomczyk, S. 2001, A\&A, 379, 622

Korycansky, D. G., \& Pringle, J. E. 1995, MNRAS, 272, 618

Lamb, H. 1909, Proc. Lond. Math. Soc., 7, 122

Lamb, H. 1932, Hydrodynamics (University Press, Cambridge), 549

Libbrecht, K. G., \& Woodard, M. F. 1991, Science, 253, 152

Nye, A. H., \& Thomas, J. H. 1974, Sol. Phys., 38, 399

Nye, A. H., \& Thomas, J. H. 1976, ApJ, 204, 573

Ogilvie, G. I., \& Lubow, S. H. 1999, ApJ, 515, 767

Roberts, B. 2001, in Encyclopedia of Astronomy and Astrophysics, ed. P. Murdin (Institute of Physics Publishing (Bristol and Philadelphia) and Nature Publishing Group (London, New York and Tokyo))

Roberts, B., \& Campbell, W. R. 1986, Nature, 323, 603

Schou, J., Antia, H. M., Basu, S., et al. 1998, ApJ, 505, 390

Schüssler, M., Caligari, P., Ferriz-Mas, A., \& Moreno-Insertis, F. 1994, A\&A, 281, L69

Slater, J. L. 1965, in Handbook of Mathematical Functions, ed. M. Abramovitz \& I. Stegun (Washington, DC: National Bureau of Standards), 503

Spiegel, E. A., \& Unno, W. 1962, PASJ, 14, 28

Vanlommel, P. 2001, Ph.D. Thesis, Katholieke Universiteit Leuven, Belgium

Woodard, M. F., \& Noyes, R. W. 1985, Nature, 318, 449

Zweibel, E. G., \& Bogdan, T. J. 1986, ApJ, 308, 401

Zweibel, E. G., \& Gough, D. O. 1995, in ESA-SP-376: Fourth SOHO Workshop Helioseismology, 73 
C. Foullon and B. Roberts: The influence of internal magnetic layers on the frequencies of solar $p$-modes, Online Material $p 1$

\section{Online Material}




\section{Appendix A: Pressures}

The distributions of the physical variables in the unperturbed configuration are denoted by a subscript o and the amplitudes of the perturbations are denoted by a hat.

The total (plasma plus magnetic) pressure

$p_{\mathrm{oT}}=p_{\mathrm{o}}+p_{\mathrm{m}}$,

obeys the equation of magnetostatic equilibrium,

$\nabla p_{\mathrm{oT}}=\rho_{\mathrm{o}} \boldsymbol{g}+\frac{1}{\mu}\left(\boldsymbol{B}_{\mathrm{o}} \cdot \nabla\right) \boldsymbol{B}_{\mathrm{o}}$

where the gradient in magnetic pressure is equal in magnitude and of opposite direction to the magnetic pressure contribution of the Lorentz force, viz.

$\nabla p_{\mathrm{m}}=\nabla \frac{B_{\mathrm{o}}^{2}}{2 \mu}$

The total pressure perturbation $\hat{p}_{\mathrm{T}}$ is

$\hat{p}_{\mathrm{T}}=\hat{p}+\frac{\boldsymbol{B}_{\mathrm{O}} \cdot \hat{\boldsymbol{B}}}{\mu}$.

Then, using the adiabatic energy equation and the induction equation to eliminate $\partial \hat{p} / \partial t$ and $\partial \hat{\boldsymbol{B}} / \partial t$,

$$
\begin{aligned}
\frac{\partial \hat{p}_{\mathrm{T}}}{\partial t} & =\frac{\partial \hat{p}}{\partial t}+\frac{\boldsymbol{B}_{\mathrm{o}}}{\mu} \cdot \frac{\partial \hat{\boldsymbol{B}}}{\partial t} \\
& =-\hat{\boldsymbol{V}} \cdot \nabla p_{\mathrm{o}}-\gamma p_{\mathrm{o}} \nabla \cdot \hat{\boldsymbol{V}}+\frac{\boldsymbol{B}_{\mathrm{o}}}{\mu} \cdot \nabla \times\left(\hat{\boldsymbol{V}} \times \boldsymbol{B}_{\mathrm{o}}\right) .
\end{aligned}
$$

Hence, the Lagrangian rate of change in total pressure perturbation $\hat{\mathcal{P}}_{\mathrm{T}}$, defined by

$\hat{\mathcal{P}}_{\mathrm{T}} \equiv \frac{D \hat{p}_{\mathrm{T}}}{D t}=\frac{\partial \hat{p}_{\mathrm{T}}}{\partial t}+\hat{\boldsymbol{V}} \cdot \nabla p_{\mathrm{oT}}$,

can be expressed (through Eq. (A.5)) as

$$
\begin{aligned}
\hat{\mathcal{P}}_{\mathrm{T}} & =\left(\frac{\partial \hat{p}_{\mathrm{T}}}{\partial t}+\hat{\boldsymbol{V}} \cdot \nabla p_{\mathrm{o}}\right)+\hat{\boldsymbol{V}} \cdot \nabla p_{\mathrm{m}} \\
& =-\gamma p_{\mathrm{o}} \nabla \cdot \hat{\boldsymbol{V}}+\frac{\boldsymbol{B}_{\mathrm{o}}}{\mu} \cdot \nabla \times\left(\hat{\boldsymbol{V}} \times \boldsymbol{B}_{\mathrm{o}}\right)+\hat{\boldsymbol{V}} \cdot \nabla p_{\mathrm{m}} \\
& =\hat{\mathcal{P}}+\hat{\mathcal{P}}_{\mathrm{m}} .
\end{aligned}
$$

Here the first term is the Lagrangian rate of change in pressure perturbation due only to the plasma, viz.

$\hat{\mathcal{P}}=-\gamma p_{\mathrm{o}} \nabla \cdot \hat{\boldsymbol{V}}$

and the second term is the component of Lagrangian rate of change in pressure perturbation due only to the magnetic field, viz.

$\hat{\mathcal{P}}_{\mathrm{m}}=\frac{1}{\mu}\left[\boldsymbol{B}_{\mathrm{o}} \cdot \nabla \times\left(\hat{\boldsymbol{V}} \times \boldsymbol{B}_{\mathrm{o}}\right)+\hat{\boldsymbol{V}} \cdot \nabla \frac{B_{\mathrm{o}}^{2}}{2}\right]$.

Using the expression for the adiabatic sound speed $c_{\mathrm{s}}(z)$, the Lagrangian rate of change in pressure perturbation due only to the plasma (A.8) can be rewritten

$\hat{\mathcal{P}}=-\gamma p_{\mathrm{o}} \hat{\Delta}=-\rho_{\mathrm{o}} c_{\mathrm{s}}^{2} \hat{\Delta}=-E \hat{\Delta}$ where $E=\rho_{\mathrm{o}} c_{\mathrm{s}}^{2}$ is the medium's modulus of elasticity and where the compressibility $\hat{\Delta}$ is defined as

$\hat{\Delta}=\nabla \cdot \hat{\boldsymbol{V}}=\frac{\partial \hat{V}_{x}}{\partial x}+\frac{\partial \hat{V}_{y}}{\partial y}+\frac{\partial \hat{V}_{z}}{\partial z}$.

For a magnetic field directed along the $x$-axis, the induced magnetic perturbation $\hat{\boldsymbol{B}}$ satisfies

$$
\begin{aligned}
\frac{\partial \hat{\boldsymbol{B}}}{\partial t} & =\nabla \times\left(\hat{\boldsymbol{V}} \times \boldsymbol{B}_{\mathrm{o}}\right) \\
& =\nabla \times\left(\hat{V}_{z} B_{\mathrm{o}} \boldsymbol{y}-\hat{V}_{y} B_{\mathrm{o}} z\right) \\
& =\left[-B_{\mathrm{o}} \frac{\partial \hat{V}_{y}}{\partial y}-\frac{\partial}{\partial z}\left(\hat{V}_{z} B_{\mathrm{o}}\right), B_{\mathrm{o}} \frac{\partial \hat{V}_{y}}{\partial x}, B_{\mathrm{o}} \frac{\partial \hat{V}_{z}}{\partial x}\right] \\
& =\left[-B_{\mathrm{o}} \hat{\Delta}_{\perp}-\hat{V}_{z} \frac{\mathrm{d} B_{\mathrm{o}}}{\mathrm{d} z}, B_{\mathrm{o}} \frac{\partial \hat{V}_{y}}{\partial x}, B_{\mathrm{o}} \frac{\partial \hat{V}_{z}}{\partial x}\right],
\end{aligned}
$$

where $\hat{\Delta}_{\perp}$ gathers the components of compressibility perpendicular to the magnetic field lines, viz.

$\hat{\Delta}_{\perp}=\frac{\partial \hat{V}_{y}}{\partial y}+\frac{\partial \hat{V}_{z}}{\partial z}$

Note that the induced magnetic perturbation shows components not only in the $x$ - and $z$-directions, but also in the $y$-direction.

The Lagrangian rate of change in pressure perturbation due only to the magnetic field (A.9) becomes

$$
\begin{aligned}
\hat{\mathcal{P}}_{\mathrm{m}} & =\frac{1}{\mu}\left(-B_{\mathrm{o}}^{2} \hat{\Delta}_{\perp}-B_{\mathrm{o}} \hat{V}_{z} \frac{\mathrm{d} B_{\mathrm{o}}}{\mathrm{d} z}+\hat{V}_{z} \frac{\mathrm{d}}{\mathrm{d} z} \frac{B_{\mathrm{o}}^{2}}{2}\right) \\
& =-\frac{B_{\mathrm{o}}^{2}}{\mu} \hat{\Delta}_{\perp}=-\rho_{\mathrm{o}} v_{\mathrm{A}}^{2} \hat{\Delta}_{\perp} .
\end{aligned}
$$

The extent to which increasing magnetic pressure decreases the volume perpendicular to the magnetic field lines is the modulus of magnetic elasticity

$E_{\mathrm{m}}=\rho_{\mathrm{o}} v_{\mathrm{A}}^{2}$.

Hence the Lagrangian rate of change in total pressure perturbation is

$$
\begin{aligned}
\hat{\mathcal{P}}_{\mathrm{T}} & =-\left(E \hat{\Delta}+E_{\mathrm{m}} \hat{\Delta}_{\perp}\right)=-\rho_{\mathrm{o}}\left(c_{\mathrm{s}}^{2} \hat{\Delta}+v_{\mathrm{A}}^{2} \hat{\Delta}_{\perp}\right) \\
& =-\rho_{\mathrm{o}} c_{\mathrm{s}}^{2}\left(1+\frac{1}{\beta} \frac{\hat{\Delta}_{\perp}}{\hat{\Delta}}\right) \hat{\Delta},
\end{aligned}
$$

and so the medium's effective modulus of elasticity is

$E_{\mathrm{T}}=\rho_{\mathrm{o}} c_{\mathrm{s}}^{2}\left(1+\frac{1}{\beta} \frac{\hat{\Delta}_{\perp}}{\hat{\Delta}}\right)=E\left(1+\frac{1}{\beta} \frac{\hat{\Delta}_{\perp}}{\hat{\Delta}}\right)$.

\section{Appendix B: Eigenfunctions}

\section{B.1. Polytropic layers}

The solution for $\Delta$ in the field-free layers are:

$\Delta(z)=\left\{\begin{array}{l}\alpha_{1} \mathrm{e}^{-k_{\mathrm{h}} z} M\left(-\Upsilon, m+2,2 k_{\mathrm{h}} z\right), \quad 0 \leq z \leq z_{\mathrm{p}}, \\ \beta_{1} \mathrm{e}^{-k_{\mathrm{h}}(z-h)} U\left(-\Upsilon, m+2,2 k_{\mathrm{h}}(z-h)\right), z \geq z_{\mathrm{q}},\end{array}\right.$ 
where $\alpha_{1}, \beta_{1}$ are arbitrary constants. It is then possible to determine the vertical velocity amplitude $V_{z}$ from Eq. (30), viz.

$V_{z}=\Delta \mathcal{D}=\frac{1}{\Omega^{4}-1}\left[\left(\frac{c_{\mathrm{s}}^{2}(z)}{g}-\frac{\gamma \Omega^{2}}{k_{\mathrm{h}}}\right) \Delta-\frac{c_{\mathrm{s}}^{2}(z) \Omega^{2}}{g k_{\mathrm{h}}} \frac{\mathrm{d} \Delta}{\mathrm{d} z}\right]$,

and the rate of change of $V_{z}$ with respect to depth (see Eq. (46))

$\frac{\mathrm{d} V_{z}}{\mathrm{~d} z}=\frac{1}{\Omega^{2}}\left[\left(\Omega^{2}-\frac{k_{\mathrm{h}} c_{\mathrm{s}}^{2}(z)}{g}\right) \Delta-k_{\mathrm{h}} V_{z}\right]$.

\section{B.2. Isothermal layer}

In the isothermal layer, the vertical velocity amplitude $V_{z}$ is given by Eq. (23). $V_{\mathrm{p}}$ and $V_{\mathrm{q}}$ are defined as the values of $V_{z}$ at depths $z=z_{\mathrm{p}}$ and $z=z_{\mathrm{q}}$, respectively:

$V_{\mathrm{p}}=V_{z}\left(z_{\mathrm{p}}\right)=D_{1} \mathrm{e}^{\Lambda^{+} z_{\mathrm{p}}}+D_{2} \mathrm{e}^{\Lambda^{-} z_{\mathrm{p}}}$

$V_{\mathrm{q}}=V_{z}\left(z_{\mathrm{q}}\right)=D_{1} \mathrm{e}^{\Lambda^{+} z_{\mathrm{q}}}+D_{2} \mathrm{e}^{\Lambda^{-} z_{\mathrm{q}}}$.

Besides Eq. (35) is verified for the eigenvalues of the dispersion relation. Hence

$D_{1} \mathcal{E}_{\mathrm{p}}^{+} \mathrm{e}^{\Lambda^{+} z_{\mathrm{p}}}+D_{2} \mathcal{E}_{\mathrm{p}}^{-} \mathrm{e}^{\Lambda^{-} z_{\mathrm{p}}}=0$

$D_{1} \mathcal{E}_{\mathrm{q}}^{+} \mathrm{e}^{\Lambda^{+} z_{\mathrm{q}}}+D_{2} \mathcal{E}_{\mathrm{q}}^{-} \mathrm{e}^{\Lambda^{-} z_{\mathrm{q}}}=0$.

In order to eliminate the constant $D_{2}$, Eqs. (B.4)-(B.7) are combined. This gives

$V_{\mathrm{p}}=D_{1} \mathrm{e}^{\Lambda^{+} z_{\mathrm{p}}}\left(1-\frac{\mathcal{E}_{\mathrm{p}}^{+}}{\mathcal{E}_{\mathrm{p}}^{-}}\right)$

$V_{\mathrm{q}}=D_{1} \mathrm{e}^{\Lambda^{+} z_{\mathrm{p}}}\left(1-\frac{\mathcal{E}_{\mathrm{q}}^{+}}{\mathcal{E}_{\mathrm{q}}^{-}}\right)$.

The constants $D_{1}$ and $D_{2}$ are obtained from Eqs. (B.8) and (B.6), giving

$V_{z}=\frac{V_{\mathrm{p}}}{1-\frac{\mathcal{E}_{\mathrm{p}}^{+}}{\mathcal{E}_{\mathrm{p}}^{-}}}\left(\mathrm{e}^{\Lambda^{+}\left(z-z_{\mathrm{p}}\right)}-\frac{\mathcal{E}_{\mathrm{p}}^{+}}{\mathcal{E}_{\mathrm{p}}^{-}} \mathrm{e}^{\Lambda^{-}\left(z-z_{\mathrm{p}}\right)}\right)$.

Hence

$\frac{\mathrm{d} V_{z}}{\mathrm{~d} z}=\frac{V_{\mathrm{p}}}{1-\frac{\mathcal{E}_{\mathrm{p}}^{+}}{\mathcal{E}_{\mathrm{p}}^{-}}}\left(\Lambda^{+} \mathrm{e}^{\Lambda^{+}\left(z-z_{\mathrm{p}}\right)}-\Lambda^{-} \frac{\mathcal{E}_{\mathrm{p}}^{+}}{\mathcal{E}_{\mathrm{p}}^{-}} \mathrm{e}^{\Lambda^{-}\left(z-z_{\mathrm{p}}\right)}\right)$,

and the ratio $\frac{1}{V_{z}} \frac{\mathrm{d} V_{z}}{\mathrm{~d} z}$ reduces to

$$
\begin{aligned}
\frac{1}{V_{z}} \frac{\mathrm{d} V_{z}}{\mathrm{~d} z} & =\frac{\Lambda^{+} \mathrm{e}^{2 \kappa\left(z-z_{\mathrm{p}}\right)}-\Lambda^{-} \frac{\mathcal{E}_{\mathrm{p}}^{+}}{\mathcal{E}_{\mathrm{p}}^{-}}}{\mathrm{e}^{2 \kappa\left(z-z_{\mathrm{p}}\right)}-\frac{\mathcal{E}_{\mathrm{p}}^{+}}{\mathcal{E}_{\mathrm{p}}^{-}}} \\
& =-\frac{1}{2 H_{\mathrm{o}}}+\kappa \frac{\mathrm{e}^{2 \kappa\left(z-z_{\mathrm{p}}\right)}+\frac{\mathcal{E}_{\mathrm{p}}^{+}}{\mathcal{E}_{\mathrm{p}}^{-}}}{\mathrm{e}^{2 \kappa\left(z-z_{\mathrm{p}}\right)}-\frac{\mathcal{E}_{\mathrm{p}}^{+}}{\mathcal{E}_{\mathrm{p}}^{-}}}
\end{aligned}
$$

\section{Appendix C: Perturbation theory}

We apply the perturbation theory for a magnetic field, as derived in Zweibel \& Gough (1995) in spherical geometry, to the problem treated here (in Cartesian geometry). Taking the azimuthal degree $m=0$ and expressing the spherical components

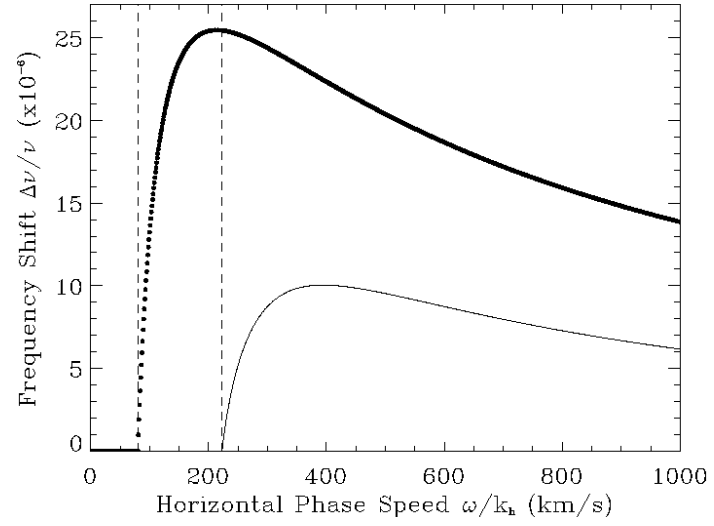

Fig. C.1. Frequency shifts $\Delta \omega / \omega$ versus the phase speed $\omega / k_{\mathrm{h}}$, obtained through a perturbation theory approach to the the shallow field and tachocline models, shown respectively in dotted (top curve) and thin (lower curve) lines. In each case, the sound speed $c_{\mathrm{c}}$ in the magnetic layer is indicated as a vertical dashed line.

for the magnetic field by $B_{r}=0$ and $B_{\theta}^{2}+B_{\phi}^{2}=B_{0}^{2}(z)$, we find that the fractional frequency change, given by Eqs. (2.4)-(2.5) in Zweibel \& Gough (1995), reduces to

$\frac{\Delta \omega}{\omega}=\oint \frac{\mathrm{d} u \delta q / c}{(\mathrm{~d} u / \mathrm{d} z)(u(1-u))^{1 / 2}} / \oint \frac{\mathrm{d} u}{(\mathrm{~d} u / \mathrm{d} z)(u(1-u))^{1 / 2}}$,

for a perturbation $\delta q$ to the sonic speed, given by

$\frac{\delta q}{c}=\frac{v_{\mathrm{A}}^{2}}{2 c_{\mathrm{c}}^{2}}(1-u)$,

and where the variable

$u=\frac{c^{2}(z)}{c^{2}\left(z_{\mathrm{t}}\right)}$

$c$ represents the phase speed or the effective wave speed at which the mode travels at the considered depth $z$, and a mode is characterised by its lower turning point $z_{\mathrm{t}}$, given when the horizontal phase speed $\omega / k_{\mathrm{h}}=c\left(z_{\mathrm{t}}\right)$.

For modes reaching the layer, we assume $c^{2}(z) \sim c_{\mathrm{S}}^{2}(z)=$ $g z / m$. In the layer, at $u=u_{\mathrm{c}}=c_{\mathrm{c}}^{2} / c^{2}\left(z_{\mathrm{t}}\right)$, the derivative $\mathrm{d} u / \mathrm{d} z=0$. Outside the layer, $\mathrm{d} u / \mathrm{d} z=g /\left(m c^{2}\left(z_{\mathrm{t}}\right)\right)$. Therefore, Eq. (C.1) becomes in this case

$\frac{\Delta \omega}{\omega} \sim \frac{v_{\mathrm{A}}^{2}}{2 c_{\mathrm{c}}^{2}} \frac{\left(1-u_{\mathrm{c}}\right)}{S\left(u_{\mathrm{c}}\left(1-u_{\mathrm{c}}\right)\right)^{1 / 2}}$,

where

$$
\begin{aligned}
S= & \frac{m c^{2}\left(z_{\mathrm{t}}\right)}{g} \int_{0}^{u_{\mathrm{c}}-\epsilon} \frac{\mathrm{d} u}{(u(1-u))^{1 / 2}} \\
& +\frac{1}{\left(u_{\mathrm{c}}\left(1-u_{\mathrm{c}}\right)\right)^{1 / 2}} \\
& +\frac{m c^{2}\left(z_{\mathrm{t}}\right)}{g} \int_{u_{\mathrm{c}}+\epsilon}^{1} \frac{\mathrm{d} u}{(u(1-u))^{1 / 2}} .
\end{aligned}
$$

Frequency shifts computed from Eqs. (C.4) and (C.5) are shown in Fig. C.1 as a function of the mode horizontal phase 
speed, for the shallow field and tachocline models. Despite differences between methods, the asymptotic solution for the tachocline model (lower curve in Fig. C.1) may be compared directly with our numerical and analytical results in Fig. 7 for parallel propagation and with our analytical results in Fig. 8 for perpendicular propagation. It appears that the perturbation theory approach reproduces the trend of the general upper envelope of the frequency shifts, with an order of magnitude higher but approaching peak values of the shifts for perpendicular propagation. The maximum amplitudes of the fractional shifts in our solutions obtained in Cartesian geometry for the tachocline model are $2 \times 10^{-6}$ and $8 \times 10^{-6}$ for respectively parallel and perpendicular propagation. Perpendicular propagation yields the largest shift, which is comparable to the maximum amplitude of the asymptotic solutions for spherical geometry $\left(10 \times 10^{-6}\right.$ in Fig. C.1). The curve for the shallow field model is also of higher magnitude (at least 2.5 times higher) than our numerical results for parallel propagation $\left(\Delta v / v=10^{-5}\right)$. The perturbation theory approach ignores the oscillatory pattern and negative extrema of the shifts and do not deal with frequency shifts from modes with turning points above the magnetic layer.
Such results can be expected given the differences between the two geometries and between the respective expressions for the perturbation to the sound speed. The asymptotic solutions to Eqs. (C.4) and (C.5) do not distinguish between parallel $\left(k_{x} \neq 0\right)$ or perpendicular $\left(k_{y} \neq 0\right)$ propagation to the magnetic field lines. They are solutions obtained in spherical geometry taking the azimuthal degree $m=0$. Moreover, the asymptotic solutions in spherical geometry are based on a perturbation for the sonic speed $\delta q / c$ given by Eq. (C.2), while our numerical results in Cartesian geometry correspond to a perturbation for the sonic speed given by Eq. (43), viz.

$$
\frac{\delta c}{c} \sim \frac{v_{\mathrm{A}}^{2}}{2 c_{\mathrm{c}}^{2}} \frac{\Delta_{\perp}}{\Delta}
$$

that is $\delta q / c$ multiplied by a correction factor. Expression (47) for $\Delta_{\perp} / \Delta$ consists of two terms: the first term corresponds to $1-u$ (by replacing $k_{x}^{2} c_{\mathrm{c}}^{2} / \omega^{2}=u$ ), and the second term is the correction factor, which involves the ratio $\frac{1}{V_{z}} \frac{\mathrm{d} V_{z}}{\mathrm{~d} z}$ at depth $z$ in the layer (Eq. (B.12)). 